\title{
The universal Euler characteristic for varieties of characteristic zero
}

\author{
Franziska Bittner
}

\begin{abstract}
Using the weak factorization theorem, we give a simple presentation for the value group of the universal Euler characteristic with compact support for varieties of characteristic zero and describe the value group of the universal Euler characteristic of pairs. This gives a new proof for the existence of natural Euler characteristics with values in the Grothendieck group of Chow motives. A generalization of the presentation to the relative setting allows us to define duality and the six operations.
\end{abstract}

\section{Introduction}

Let $k$ be a field of characteristic zero. For $A$ an abelian group, an $A$-valued Euler characteristic with compact support for varieties over $k$ assigns to every such variety $X$ an element $e(X) \in A$ only depending on the isomorphism class of $X$ such that $e(X)=e(X-Y)+e(Y)$ for $Y \subset X$ a closed subvariety. There is an evident universal Euler characteristic for varieties. It takes values in the naive Grothendieck group of varieties over $k$, denoted by $\mathrm{K}_{0}\left(\operatorname{Var}_{k}\right)$, which is the free abelian group on isomorphism classes $[X]$ of varieties $X$ over $k$ modulo the relations $[X]=[X-Y]+[Y]$ for $Y \subset X$ a closed subvariety. It can be given a ring structure by taking products of varieties. This group and its relative and equivariant analogues (to be more precise, certain localizations and completions) are the natural value groups for motivic integrals, as explained, e.g., by Looijenga in [Loo02] and by Denef and Loeser in [DL01].

In this note we give a simple presentation of this group in terms of smooth projective varieties. In fact we show that $\mathrm{K}_{0}\left(\operatorname{Var}_{k}\right)$ is the free abelian group on isomorphism classes of smooth projective varieties modulo the relations $[\emptyset]=0$ and $[X]-[Y]=\left[\mathrm{Bl}_{Y} X\right]-[E]$, where $Y \subset X$ is a smooth closed subvariety, $\mathrm{Bl}_{Y} X$ denotes the blow-up of $X$ along $Y$ and $E$ denotes the exceptional divisor of this blow-up. The key ingredient is the factorization theorem proven by Włodarczyk in [Wło03] and Abramovich et al. in [AKMW02]: Any proper birational map between smooth irreducible varieties over $k$ can be factored into a sequence of blow-ups and blow-downs with smooth centers. It follows that there is a unique Euler characteristic with compact support for $k$-varieties with values in the Grothendieck group of Chow motives over $k$ which assigns to a smooth projective variety the class of its Chow motive. This has already been proven by Gillet and Soulé in [GS96] and by Guillen and Navarro Aznar in [GNA02]. Also a result by Larsen and Lunts in [LL03] follows.

There is a similar, though somewhat more involved, definition of an Euler characteristic of pairs of varieties. We show that the universal Euler characteristic for pairs has the same value ring as the universal Euler characteristic with compact support, hence for every pair $(X, Y)$ we get an element $\langle X, Y\rangle$ in $\mathrm{K}_{0}\left(\operatorname{Var}_{k}\right)$. In these terms $[X]$ corresponds to $\langle\bar{X}, \bar{X}-X\rangle$, where $X \subset \bar{X}$ is a completion of $X$.

Received 29 March 2002, accepted in final form 5 August 2002.

2000 Mathematics Subject Classification 14E05, 14F42.

Keywords: Grothendieck groups of varieties, Euler characteristic, weak factorization.

This journal is (C) Foundation Compositio Mathematica 2004. 


\section{F. BITTNER}

We are then in a position to show that there is a unique Euler characteristic for pairs of $k$-varieties with values in the Grothendieck group of Chow motives which for a smooth projective variety $X$ assigns to the pair $(X, \emptyset)$ the Chow motive of $X$. This gives a new proof for a result by Guillen and Navarro Aznar in [GNA02].

We also develop simple presentations for the Grothendieck group of varieties over a base variety since this will make the theory much more effective. For instance, we are then able to bring in the operations $f_{*}, f_{!}, f^{*}, f^{!}$, tensor product, internal homomorphisms and duality familiar from Grothendieck's duality theory and derive some expected formulae.

Furthermore we generalize the presentations and operations to an equivariant setting.

Conventions. In the following, $k$ denotes a field of characteristic zero. By a variety over $k$ we mean a reduced separated scheme of finite type over $k$, not necessarily irreducible.

\section{The weak factorization theorem}

The weak factorization theorem for varieties over a (not necessarily algebraically closed) field $k$ of characteristic zero is proven by Włodarczyk in [Wło03] and Abramovich et al. in [AKMW02]. For convenience we recall parts of Theorem 0.3.1 in [AKMW02] and the subsequent Remark 2.

Theorem 2.1. Let $\phi: X_{1} \rightarrow X_{2}$ be a birational map between complete smooth connected varieties over $k$, and let $U \subset X_{1}$ be an open set where $\phi$ is an isomorphism. Then $\phi$ can be factored into a sequence of blow-ups and blow-downs with smooth centers disjoint from $U$ : there exists a sequence of birational maps

$$
X_{1}=V_{0} \stackrel{\phi_{1}}{\rightarrow} V_{1} \stackrel{\phi_{2}}{\rightarrow} \cdots \stackrel{\phi_{i}}{\rightarrow} V_{i} \stackrel{\phi_{i+1}}{\rightarrow} V_{i+1} \stackrel{\phi_{i+2}}{\rightarrow} \cdots \stackrel{\phi_{l-1}}{\rightarrow} V_{l-1} \stackrel{\phi_{l}}{\rightarrow} V_{l}=X_{2},
$$

where $\phi=\phi_{l} \circ \phi_{l-1} \circ \cdots \phi_{2} \circ \phi_{1}$, such that each factor $\phi_{i}$ is an isomorphism over $U$, and $\phi_{i}: V_{i}-\rightarrow V_{i+1}$ or $\phi_{i}^{-1}: V_{i+1} \rightarrow V_{i}$ is a morphism obtained by blowing up a smooth center disjoint from $U$ (here $U$ is identified with an open subset of $V_{i}$ ).

Moreover, there is an index $i_{0}$ such that for all $i \leqslant i_{0}$ the map $V_{i} \rightarrow X_{1}$ is defined everywhere and projective, and for all $i \geqslant i_{0}$ the map $V_{i} \rightarrow X_{2}$ is defined everywhere and projective.

If $X_{1}-U$ (respectively, $X_{2}-U$ ) is a simple normal crossings divisor, then the factorization can be chosen such that the inverse images of this divisor under $V_{i} \rightarrow X_{1}$ (respectively, $V_{i} \rightarrow X_{2}$ ) are also simple normal crossings divisors, and the centers of blowing up have normal crossings with these divisors.

If $\phi$ is equivariant under the action of a finite group (i.e. the graph of $\phi$ is invariant under the diagonal $G$-action), then the factorization can be chosen equivariantly.

We make some remarks on this theorem.

Remark 2.2. It is not explicitly stated in Theorem 0.3.1 of [AKMW02] that, in the case of $X_{1}-U$ (respectively, $X_{2}-U$ ) a simple normal crossings divisor, the inverse images of this divisor under $V_{i} \rightarrow X_{1}$ (respectively, $V_{i} \rightarrow X_{2}$ ) are also simple normal crossings divisors, but it can be read off from the proof (see 5.9 and 5.10 in [AKMW02]).

Remark 2.3. The completeness of the varieties $X_{1}$ and $X_{2}$ is not necessary as has already been pointed out by Bonavero in [Bon02]. It suffices that $\phi$ is a proper birational map $X_{1} \rightarrow X_{2}$ between smooth connected varieties which is an isomorphism on an open subset $U \subset X_{1}$. This means that the projections to $X_{1}$ and $X_{2}$ from the graph of $\phi$ are proper, which gives back usual properness for an everywhere defined $\phi$. 


\section{The universal Euler CHARACTERISTiC}

Remark 2.4. We note that the theorem implies that if $X_{1}$ and $X_{2}$ are varieties over a base variety $S$ and $\phi$ is a map of $S$-varieties then the factorization is a factorization over $S$. If $X_{1}$ and $X_{2}$ are projective over $S$ then so are the $V_{i}$.

Definition 2.5. An action of a finite group on a variety is said to be good if every orbit is contained in an affine open set.

Remark 2.6. Note that on a quasi-projective variety every action of a finite group is good. More generally, if $X \longrightarrow S$ is equivariant and $X$ is quasi-projective over $S$, then the action on $X$ is good if the action on $S$ is. In particular, in the case of an equivariant factorization with respect to a good action of a finite group on $X_{1}$ and $X_{2}$ the action on the $V_{i}$ is automatically good as they are projective over $X_{1}$, or $X_{2}$ respectively.

Remark 2.7. If $X$ is a not necessarily connected variety with a (good) $G$-action such that $G$ acts transitively on the connected components of $X$, one can identify $X$ with the induced variety $G \times{ }^{G_{0}}$ $X_{0}=\left(G \times X_{0}\right) / G_{0}$, where $X_{0}$ is a connected component of $X$ and $G_{0}$ is the stabilizer of $X_{0}$. Here the action of $G_{0}$ is given by $h(g, x)=\left(g h^{-1}, h x\right)$.

This, together with the equivariant version of the factorization theorem for connected smooth varieties, also yields an equivariant factorization for smooth, not necessarily connected varieties, provided $G$ acts transitively on the connected components.

\section{The universal Euler characteristic with compact support}

Let $\mathrm{K}_{0}\left(\operatorname{Var}_{k}\right)$ be the free abelian group on isomorphism classes $[X]$ of varieties $X$ over $k$ where we impose the relation $[X]=[X-Y]+[Y]$ for $Y \subset X$ a closed subvariety (so in particular $[\emptyset]=0$ ). This group is also called the (naive) Grothendieck group of k-varieties. It is the value group of the universal Euler characteristic with compact support. It is a commutative ring for the multiplication induced by the product of $k$-varieties, because the subgroup divided out actually is an ideal with respect to this multiplication.

Theorem 3.1. The Grothendieck group of $k$-varieties has the following alternative presentations:

$\mathrm{sm})$ as the abelian group generated by the isomorphism classes of smooth varieties over $k$ subject to the relations $[X]=[X-Y]+[Y]$, where $X$ is smooth and $Y \subset X$ is a smooth closed subvariety;

bl) as the abelian group generated by the isomorphism classes of smooth complete $k$-varieties subject to the relations $[\emptyset]=0$ and $\left[\mathrm{Bl}_{Y} X\right]-[E]=[X]-[Y]$, where $X$ is smooth and complete, $Y \subset X$ is a closed smooth subvariety, $\mathrm{Bl}_{Y} X$ is the blow-up of $X$ along $Y$ and $E$ is the exceptional divisor of this blow-up.

Moreover, we get the same group if in case ( $\mathrm{sm}$ ) we restrict to quasi-projective varieties or if in case (bl) we restrict to projective varieties. We can also restrict to connected varieties in both presentations.

Remark 3.2. The subgroups divided out are again ideals with respect to the product of $k$-varieties.

Definition 3.3. Let $\mathbb{L}=\left[\mathbb{A}^{1}\right]$ denote the class of the affine line. Define the naive motivic ring of $k$-varieties, $\mathcal{M}_{k}$, as the localization $\mathrm{K}_{0}\left(\operatorname{Var}_{k}\right)\left[\mathbb{L}^{-1}\right]$.

COROLlary 3.4. We have a ring involution $\mathcal{D}_{k}$ of $\mathcal{M}_{k}$ that sends $\mathbb{L}$ to $\mathbb{L}^{-1}$ and is characterized by the property that it sends the class of a complete connected smooth variety $X$ to $\mathbb{L}^{-\operatorname{dim} X}[X]$.

We call this involution the duality map. 


\section{F. BITTNER}

We first deduce Corollary 3.4 from Theorem 3.1.

A vector bundle $V \longrightarrow X$ of rank $n$ on a variety $X$ is locally trivial by definition and hence its class $[V]$ in $\mathrm{K}_{0}\left(\operatorname{Var}_{k}\right)$ is equal to $\mathbb{L}^{n}[X]$. Similarly the class of its projectivization $[\mathbb{P}(V)]$ equals $\left[\mathbb{P}^{n-1}\right][X]=\left(1+\mathbb{L}+\cdots+\mathbb{L}^{n-1}\right)[X]$.

Lemma 3.5. Let $X$ be a smooth connected variety and $Y \subset X$ a smooth connected subvariety, and let $d$ denote the codimension of $Y$ in $X$. Let $E$ be the exceptional divisor of the blow-up $\mathrm{Bl}_{Y} X$ of $X$ along $Y$. Then $\left[\mathrm{Bl}_{Y} X\right]-\mathbb{L}[E]=[X]-\mathbb{L}^{d}[Y]$ in $\mathrm{K}_{0}\left(\operatorname{Var}_{k}\right)$.

Proof. In $\mathrm{K}_{0}\left(\operatorname{Var}_{k}\right)$ we have the fundamental relation $\left[\mathrm{Bl}_{Y} X\right]-[E]=[X]-[Y]$. Furthermore $[E]=\left(1+\mathbb{L}+\cdots+\mathbb{L}^{d-1}\right)[Y]$, thus $(1-\mathbb{L})[E]=\left(1-\mathbb{L}^{d}\right)[Y]$. Adding this to the fundamental relation finishes the proof.

Proof of Corollary 3.4. Using the presentation (bl) and Lemma 3.5 we can define a group homomorphism $\mathrm{K}_{0}\left(\operatorname{Var}_{k}\right) \longrightarrow \mathcal{M}_{k}$ by sending the class of a smooth connected complete variety $X$ to $\mathbb{L}^{-\operatorname{dim} X}[X]$. This morphism is multiplicative and maps $\mathbb{L}$ to $\mathbb{L}^{-1}$, hence it can be extended uniquely to a ring endomorphism $\mathcal{D}_{k}$ of $\mathcal{M}_{k}$. Obviously $\mathcal{D}_{k} \mathcal{D}_{k}=\mathrm{id}_{\mathcal{M}_{k}}$.

We now deduce the presentation $(\mathrm{sm})$ of Theorem 3.1. For this purpose let us provisionally introduce the group $\mathrm{K}_{0}^{\mathrm{sm}}\left(\operatorname{Var}_{k}\right)$, defined as the free abelian group on isomorphism classes $[X]_{\mathrm{sm}}$ of smooth varieties modulo the relations for smooth closed subvarieties $Y$ of smooth varieties $X$. It is a commutative ring with respect to the product of varieties.

Step 1. The ring homomorphism $\mathrm{K}_{0}^{\mathrm{sm}}\left(\operatorname{Var}_{k}\right) \longrightarrow \mathrm{K}_{0}\left(\operatorname{Var}_{k}\right),[X]_{\mathrm{sm}} \mapsto[X]$ is an isomorphism.

Proof. To construct an inverse for any variety $X$ we stratify $X=\bigsqcup_{N \in \mathcal{N}} N$ such that $N$ is smooth and equidimensional and $\bar{N}$ is a union of strata for all $N \in \mathcal{N}$. Consider the expression $\sum_{N \in \mathcal{N}}[N]_{\mathrm{sm}}$ in $\mathrm{K}_{0}^{\mathrm{sm}}\left(\operatorname{Var}_{k}\right)$. If $X$ is smooth itself $\sum_{N \in \mathcal{N}}[N]_{\mathrm{sm}}$ equals $[X]_{\mathrm{sm}}$ as can be seen by induction on the number of elements of $\mathcal{N}$ : Let $N \in \mathcal{N}$ be an element of minimal dimension, then $[X]_{\mathrm{sm}}=$ $[X-N]_{\mathrm{sm}}+[N]_{\mathrm{sm}}$, and by the induction hypothesis $[X-N]_{\mathrm{sm}}=\sum_{N^{\prime} \in \mathcal{N}-\{N\}}\left[N^{\prime}\right]_{\mathrm{sm}}$.

For two stratifications $\mathcal{N}$ and $\mathcal{N}^{\prime}$ of $X$ we can always find a common refinement $\mathcal{L}$. The above argument shows that for $N \in \mathcal{N}$ we get $\sum_{N \supset L \in \mathcal{L}}[L]_{\mathrm{sm}}=[N]_{\mathrm{sm}}$. Hence $\sum_{L \in \mathcal{L}}[L]_{\mathrm{sm}}$ is equal to $\sum_{N \in \mathcal{N}}[N]_{\mathrm{sm}}$ and analogously it equals $\sum_{N \in \mathcal{N}^{\prime}}[N]_{\mathrm{sm}}$, therefore $\sum_{N \in \mathcal{N}}[N]_{\mathrm{sm}}$ is independent of the choice of the stratification. Thus we can set $e(X):=\sum_{N \in \mathcal{N}}[N]_{\mathrm{sm}}$.

If $Y \subset X$ is a closed subvariety we can find a stratification for which $Y$ is a union of strata which yields $e(X)=e(X-Y)+e(Y)$, hence $e$ is an Euler characteristic with compact support and factors through $\mathrm{K}_{0}\left(\operatorname{Var}_{k}\right)$. The induced map on $\mathrm{K}_{0}\left(\operatorname{Var}_{k}\right)$ obviously is an inverse for $\mathrm{K}_{0}^{\mathrm{sm}}\left(\operatorname{Var}_{k}\right) \longrightarrow$ $\mathrm{K}_{0}\left(\operatorname{Var}_{k}\right)$.

Decomposing into connected components, and noting that instead of cutting a smooth closed subvariety $Y$ out of a smooth connected variety $X$ we can also take out the connected components of $Y$ one by one, shows that we can restrict to smooth connected varieties in the presentation (sm).

Stratifying by smooth quasi-projective varieties shows that we can restrict to smooth (connected) quasi-projective varieties.

In the rest of this section we establish the presentation (bl) of Theorem 3.1.

Again we introduce an auxiliary group: Let $K_{0}^{\mathrm{bl}}\left(\operatorname{Var}_{k}\right)$ be the free abelian group on isomorphism classes $[X]_{\mathrm{bl}}$ of smooth complete varieties $X$ over $k$ modulo the relations for blow-ups of smooth complete varieties $X$ along smooth closed subvarieties $Y$ and the relation $[\emptyset]_{\mathrm{bl}}=0$ (then $[X \sqcup Y]_{\mathrm{bl}}=$ $[X]_{\mathrm{bl}}+[Y]_{\mathrm{bl}}$, which can be seen by blowing up along $\left.Y\right)$.

Decomposing into connected components and noting that the blow-up along a disjoint union is the successive blow-up along the connected components, one sees that this can also be described as 


\section{The universal Euler CHARACTERISTiC}

the free abelian group on isomorphism classes $[X]_{\mathrm{bl}}$ of connected smooth complete varieties with imposed relations $[\emptyset]_{\mathrm{bl}}=0$ and $\left[\mathrm{Bl}_{Y} X\right]_{\mathrm{bl}}-[E]_{\mathrm{bl}}=[X]_{\mathrm{bl}}-[Y]_{\mathrm{bl}}$, where $Y \subset X$ is a connected closed smooth subvariety.

Also $\mathrm{K}_{0}^{\mathrm{bl}}\left(\operatorname{Var}_{k}\right)$ carries a commutative ring structure induced by the product of varieties.

Step 2. The ring homomorphism $\mathrm{K}_{0}^{\mathrm{bl}}\left(\operatorname{Var}_{k}\right) \longrightarrow \mathrm{K}_{0}\left(\operatorname{Var}_{k}\right)$ which sends $[X]_{\mathrm{bl}}$ to $[X]$ is an isomorphism.

Proof. Again we construct an inverse. Using the presentation (sm) in Theorem 3.1 we see that defining an Euler characteristic with compact support is equivalent to defining an Euler characteristic with compact support for smooth connected varieties.

Let $X$ be a smooth connected variety of dimension $n$, and let $X \subset \bar{X}$ be a smooth completion with $D=\bar{X}-X$ a simple normal crossings divisor. Let $D^{(l)}$ be the normalization of the $l$-fold intersections of $D$, where $D^{(0)}$ is understood to be $\bar{X}$ (so $D^{(l)}$ is the disjoint union of the $l$-fold intersections of the irreducible components of $D)$. Consider the expression $\sum_{l=0}^{n}(-1)^{l}\left[D^{(l)}\right]_{\mathrm{bl}}$ in $\mathrm{K}_{0}^{\mathrm{bl}}\left(\operatorname{Var}_{k}\right)$.

We first show that this expression is independent of the choice of the completion:

Let $X \subset \bar{X}^{\prime}$ and $X \subset \bar{X}$ be two smooth completions of $X$ with $\bar{X}-X=D$ and $\bar{X}^{\prime}-X=D^{\prime}$ simple normal crossings divisors. Owing to the weak factorization theorem the birational map $\bar{X}^{\prime} \rightarrow \bar{X}$ can be factored into a sequence of blow-ups and blow-downs between smooth complete varieties with smooth centers disjoint from $X$ which have normal crossings with the complement of $X$. Hence we may assume that $\bar{X}^{\prime}=\mathrm{Bl}_{Z} \bar{X}$ with $Z \subset D$ smooth and connected such that $Z$ has normal crossings with $D$.

Let $D_{0}$ be an irreducible component of $D$ containing $Z$ and let $\left\{D_{i}\right\}_{i \in I}$ be the remaining irreducible components. Then the irreducible components of $D^{\prime}$ are $D_{i}^{\prime}=\operatorname{Bl}_{Z \cap D_{i}} D_{i}$ (where $i \in\{0\} \cup I$ ) and the exceptional divisor $E$ of the blow-up. For $K \subset\{0\} \cup I$ we put $D_{K}:=\bigcap_{j \in K} D_{j}$ (where $D_{\emptyset}$ is understood to be $\bar{X}), D_{K}^{\prime}:=\bigcap_{j \in K} D_{j}^{\prime}, Z_{K}:=Z \cap D_{K}$ and $E_{K}:=E \cap D_{K}^{\prime}$. As $Z$ has simple normal crossings with $D$ we get $D_{K}^{\prime}=\mathrm{Bl}_{Z_{K}} D_{K}$ with exceptional divisor $E_{K}$, hence we have

$$
\left[D_{K}^{\prime}\right]_{\mathrm{bl}}-\left[E_{K}\right]_{\mathrm{bl}}=\left[D_{K}\right]_{\mathrm{bl}}-\left[Z_{K}\right]_{\mathrm{bl}} \text {. }
$$

Denote by $E^{(l)}$ the preimage of $E$ in $D^{\prime(l)}$ and by $Z^{(l)}$ the preimage of $Z$ in $D^{(l)}$. Then for $l=0, \ldots, n$ the preceding identity yields

$$
\begin{aligned}
{\left[D^{\prime(l)}\right]_{\mathrm{bl}} } & =\sum_{|K|=l}\left[D_{K}^{\prime}\right]_{\mathrm{bl}}+\sum_{|K|=l-1}\left[E_{K}\right]_{\mathrm{bl}} \\
& =\sum_{|K|=l}\left(\left[D_{K}\right]_{\mathrm{bl}}+\left[E_{K}\right]_{\mathrm{bl}}-\left[Z_{K}\right]_{\mathrm{bl}}\right)+\sum_{|K|=l-1}\left[E_{K}\right]_{\mathrm{bl}} \\
& =\left[D^{(l)}\right]_{\mathrm{bl}}+\left[E^{(l)}\right]_{\mathrm{bl}}-\left[Z^{(l)}\right]_{\mathrm{bl}}+\left[E^{(l-1)}\right]_{\mathrm{bl}}
\end{aligned}
$$

(for $l=0$ the last term is zero). As $Z \subset D_{0}$ we get $Z_{\{0\} \cup K}=Z_{K}$ for $K \subset I$, thus $\sum(-1)^{l}\left[Z^{(l)}\right]_{\mathrm{bl}}=0$. Taking the alternating sum hence yields

$$
\sum(-1)^{l}\left[D^{\prime(l)}\right]_{\mathrm{bl}}=\sum(-1)^{l}\left[D^{(l)}\right]_{\mathrm{bl}}
$$

Therefore we can set $e(X):=\sum(-1)^{l}\left[D^{(l)}\right]_{\mathrm{bl}}$.

We have to check that $e(X)=e(X-Y)+e(Y)$ for $Y \subset X$ a connected closed smooth subvariety of a connected smooth variety $X$. We choose $\bar{X} \supset X$ smooth and complete such that $D=\bar{X}-X$ is a simple normal crossings divisor and such that the closure $\bar{Y}$ of $Y$ in $\bar{X}$ is also smooth and has normal crossings with $D$ (we can take first a smooth completion of $X$ with boundary a simple normal crossings divisor and then an embedded resolution of the closure of $Y$ compatible with this 


\section{F. BitTneR}

divisor - compare, e.g., $\S 1.2$ of [AKMW02]). In particular $D \cap \bar{Y}$ is a simple normal crossings divisor in $\bar{Y}$. Denote the irreducible components of $D$ by $\left\{D_{i}\right\}_{i \in I}$, for $K \subset I$ let $D_{K}$ be defined as above, let $Y_{K}:=\bar{Y} \cap D_{K}$ and $Y^{(l)}=\bigsqcup_{|K|=l} Y_{K}$. Then $e(Y)=\sum(-1)^{l}\left[Y^{(l)}\right]_{\mathrm{bl}}$.

Let $\widetilde{X}:=\mathrm{Bl}_{\bar{Y}} \bar{X}$ and denote the exceptional divisor by $E$. Denote the proper transform of $D_{i}$ by $\widetilde{D}_{i}$. The complement $\widetilde{D}$ of $X-Y$ in $\widetilde{X}$ is the simple normal crossings divisor $\cup \widetilde{D}_{i} \cup E$. If $E_{K}:=E \cap \widetilde{D}_{K}$ then as above $\widetilde{D}_{K}$ is the blow-up of $D_{K}$ along $Y_{K}$ with exceptional divisor $E_{K}$. Thus

$$
\begin{aligned}
{\left[\widetilde{D}^{(l)}\right]_{\mathrm{bl}} } & =\sum_{|K|=l}\left[\widetilde{D}_{K}\right]_{\mathrm{bl}}+\sum_{|K|=l-1}\left[E_{K}\right]_{\mathrm{bl}} \\
& =\sum_{|K|=l}\left(\left[D_{K}\right]_{\mathrm{bl}}-\left[Y_{K}\right]_{\mathrm{bl}}+\left[E_{K}\right]_{\mathrm{bl}}\right)+\sum_{|K|=l-1}\left[E_{K}\right]_{\mathrm{bl}} \\
& =\left[D^{(l)}\right]_{\mathrm{bl}}-\left[Y^{(l)}\right]_{\mathrm{bl}}+\left[E^{(l)}\right]_{\mathrm{bl}}+\left[E^{(l-1)}\right]_{\mathrm{bl}} .
\end{aligned}
$$

Taking the alternating sum yields $e(X-Y)=e(X)-e(Y)$. Hence $e$ induces a morphism $\mathrm{K}_{0}\left(\operatorname{Var}_{k}\right)$ $\longrightarrow \mathrm{K}_{0}^{\mathrm{bl}}\left(\operatorname{Var}_{k}\right)$ which clearly is an inverse for the mapping $\mathrm{K}_{0}^{\mathrm{bl}}\left(\operatorname{Var}_{k}\right) \longrightarrow \mathrm{K}_{0}\left(\operatorname{Var}_{k}\right)$.

Using the fact that we can restrict to quasi-projective generators in the presentation (sm) of Theorem 3.1 and that a connected smooth quasi-projective variety has a smooth projective simple normal crossings completion, we see that we can restrict to projective generators in the description (bl) of Theorem 3.1.

\section{The universal Euler characteristic of pairs}

Let $A$ be an abelian group. Then an $A$-valued Euler characteristic is a mapping which associates to each pair $(X, Y)$ of varieties over $k$ with $Y \subset X$ a closed subvariety an element $\chi(X, Y) \in A$ (where we denote $\chi(X, \emptyset)$ by $\chi(X))$ such that the following properties hold.

- Additivity. If $X \supset_{\text {closed }} Y \supset_{\text {closed }} Z$, then $\chi(X, Z)=\chi(X, Y)+\chi(Y, Z)$.

- Excision. If $f: X^{\prime} \longrightarrow X$ is proper and $Y \subset X$ is a closed subvariety such that $f$ induces an isomorphism $X^{\prime}-f^{-1} Y \cong X-Y$, then $\chi\left(X^{\prime}, f^{-1} Y\right)=\chi(X, Y)$.

- Gysin. If $X$ is smooth and connected and $D \subset X$ is a smooth divisor, then $\chi(X-D)=$ $\chi(X)-\chi\left(\mathbb{P}^{1} \times D,\{\infty\} \times D\right)$.

Excision implies that $\chi(X, Y)$ only depends on the isomorphism class of the pair $(X, Y)$. Using additivity we get $\chi(X, Y)=\chi(X)-\chi(Y)$. Additivity and excision yield $\chi(X \sqcup Y)=\chi(X \sqcup Y, Y)+$ $\chi(Y)=\chi(X)+\chi(Y)$ and $\chi(\emptyset)=0$. If $X \subset W$ is an open embedding with $W$ complete, then the excision property implies that $\chi(W, W-X)$ is independent of the choice of the open embedding. We denote $\chi(W, W-X)$ by $\chi_{c}(X)$. For $Y \subset X$ closed we have

$$
\begin{aligned}
\chi_{c}(X) & =\chi(W, W-X) \\
& =\chi(W, \bar{Y} \cup(W-X))+\chi(\bar{Y} \cup(W-X), W-X) \\
& =\chi_{c}(X-Y)+\chi_{c}(Y),
\end{aligned}
$$

where by $\bar{Y}$ we denote the closure of $Y$ in $W$. Hence $\chi_{c}$ factors through $\mathrm{K}_{0}\left(\operatorname{Var}_{k}\right)$.

DEFINITION 4.1. The value ring of the universal Euler characteristic for pairs of $k$-varieties is defined to be the free abelian group on isomorphism classes of pairs $(X, Y)$ with $Y \subset X$ closed modulo the relations for an Euler characteristic. We denote it by $\widetilde{\mathrm{K}}_{0}\left(\operatorname{Var}_{k}\right)$. The class of a pair $(X, Y)$ in $\widetilde{\mathrm{K}}_{0}\left(\operatorname{Var}_{k}\right)$ is denoted by $\langle X, Y\rangle$, where we write $\langle X\rangle$ as shorthand for $\langle X, \emptyset\rangle$. The product of $\langle X, Y\rangle$ and $\left\langle X^{\prime}, Y^{\prime}\right\rangle$ is defined to be $\left\langle X \times X^{\prime}, X \times Y^{\prime} \cup Y \times X^{\prime}\right\rangle$. 


\section{The universal Euler CHARACTERISTiC}

The above construction yields a group homomorphism

$$
\chi_{c}: \mathrm{K}_{0}\left(\operatorname{Var}_{k}\right) \longrightarrow \widetilde{\mathrm{K}}_{0}\left(\operatorname{Var}_{k}\right),
$$

which is actually a ring homomorphism.

To see that the ring structure on $\widetilde{\mathrm{K}}_{0}\left(\operatorname{Var}_{k}\right)$ is compatible with the Gysin relation, we introduce an auxiliary group $\widehat{\mathrm{K}_{0}}\left(\operatorname{Var}_{k}\right)$, defined as the free abelian group on isomorphism classes of pairs of varieties over $k$ modulo excision and additivity. We denote the class of a pair $(X, Y)$ in $\widehat{\mathrm{K}_{0}}\left(\operatorname{Var}_{k}\right)$ by $\{X, Y\}$, where we write $\{X\}$ for $\{X, \emptyset\}$. We get a ring structure on $\widehat{\mathrm{K}_{0}}\left(\operatorname{Var}_{k}\right)$ by setting $\{X, Y\}\left\{X^{\prime}, Y^{\prime}\right\}=\left\{X \times X^{\prime}, X \times Y^{\prime} \cup Y \times X^{\prime}\right\}$. Noting that $\{X, Y\}=\{X\}-\{Y\}$ and $\{X, S\}=\{\widetilde{X}, \widetilde{S}\}$ for an arbitrary $X$, where $S$ is the singular locus of $X$ and $\widetilde{X}$ a resolution of singularities with $\widetilde{S}$ the inverse image of $S$, we get $\{X\}=\{\widetilde{X}\}-\{\widetilde{S}\}+\{S\}$, where the dimension of $\widetilde{S}$ and $S$ is strictly less than the dimension of $X$. Proceeding inductively on the dimension of $X$ we can write every class $\{X, Y\}$ as a linear combination of classes of smooth varieties. Hence the subgroup generated by expressions of the form $\{X-D\}-\{X\}+\left\{\mathbb{P}^{1} \times D,\{\infty\} \times D\right\}$ with $X$ smooth and $D$ a smooth divisor on $X$ actually is an ideal.

Using the multiplicative structure of $\widetilde{\mathrm{K}}_{0}\left(\operatorname{Var}_{k}\right)$ the Gysin relation can be rewritten as

$$
\langle X-D\rangle=\langle X\rangle-\left\langle\mathbb{P}^{1},\{\infty\}\right\rangle\langle D\rangle \text {. }
$$

Theorem 4.2. The map $\chi_{c}: \mathrm{K}_{0}\left(\operatorname{Var}_{k}\right) \longrightarrow \widetilde{\mathrm{K}}_{0}\left(\operatorname{Var}_{k}\right)$ is a ring isomorphism. If $X$ is a smooth connected $k$-variety and $(\bar{X}, D)$ is a simple normal crossings completion of $X$ over $k$, then the inverse of $\chi_{c}$ assigns to $\langle X\rangle$ the element $\sum(-\mathbb{L})^{l}\left[D^{(l)}\right]$. Its image in $\mathcal{M}_{k}$ is also equal to $\mathcal{D}_{k}\left(\mathbb{L}^{-\operatorname{dim} X}[X]\right)=$ $\mathbb{L}^{\operatorname{dim} X} \mathcal{D}_{k}([X])$.

Before we prove this we give an application of Theorems 3.1 and 4.2.

Let $\mathfrak{M}_{k}$ denote the category of Chow $k$-motives (see e.g. the introduction [Sch94] by Scholl for the definition of $\left.\mathfrak{M}_{k}\right)$. For a smooth connected projective variety $X$ over $k$, denote by $h(X)$ the motive of $X$ and its class in $\mathrm{K}_{0}\left(\mathfrak{M}_{k}\right)$ by $[h(X)]$. Denote the class $\left[h\left(\mathbb{P}^{1}\right)\right]-[h($ Spec $k)]$ of the Tate motive by $\mathbb{L}_{\text {mot }}$, and by $[h(X)]^{\vee}=\mathbb{L}_{\text {mot }}^{-\operatorname{dim} X} \otimes[h(X)]$ the class of the dual motive of $h(X)$.

COROLlary 4.3. There is a ring homomorphism (in fact the unique group homomorphism) $\chi_{c, \text { mot }}$ : $\mathrm{K}_{0}\left(\operatorname{Var}_{k}\right) \longrightarrow \mathrm{K}_{0}\left(\mathfrak{M}_{k}\right)$ which sends the class of a smooth projective variety $X$ to $[h(X)]$. The class of the affine line is mapped to the class of the Tate motive by this morphism. Likewise, there is a ring homomorphism (in fact the unique group homomorphism) $\chi_{\operatorname{mot}}: \widetilde{\mathrm{K}}_{0}\left(\operatorname{Var}_{k}\right) \longrightarrow \mathrm{K}_{0}\left(\mathfrak{M}_{k}\right)$ which sends the class of a smooth projective variety $X$ to $[h(X)]$. The two are related by the property that for $X$ smooth and connected we have $\left(\chi_{\operatorname{mot}}\langle X\rangle\right)^{\vee}=\mathbb{L}_{\operatorname{mot}}^{-\operatorname{dim} X} \otimes \chi_{c, \operatorname{mot}}[X]$.

Proof of Corollary 4.3. For $Y \subset X$ a smooth closed subvariety of a smooth projective variety we have $\left[h\left(\mathrm{Bl}_{Y} X\right)\right]-[h(E)]=[h(X)]-[h(Y)]$, where $E$ denotes the exceptional divisor. We now use the presentation (bl) of Theorem 3.1 to see that there is a unique group homomorphism $\mathrm{K}_{0}\left(\operatorname{Var}_{k}\right) \longrightarrow$ $\mathrm{K}_{0}\left(\mathfrak{M}_{k}\right)$ which sends the class of a smooth projective variety $X$ to $[h(X)]$ and therefore is a ring homomorphism. As $\mathbb{L}$ is mapped to $\mathbb{L}_{\text {mot }}$ it can be extended to $\mathcal{M}_{k}$.

Furthermore due to Theorem 4.2 there is a unique group homomorphism $\chi_{\text {mot }}$ (in fact a ring homomorphism) which makes

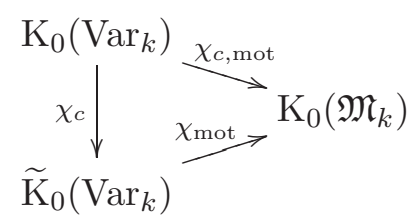

commutative. 


\section{F. BitTneR}

Now let $X$ be a smooth connected variety. As the image of $\chi_{c}^{-1}\langle X\rangle$ in $\mathcal{M}_{k}$ is $\mathcal{D}_{k}\left(\mathbb{L}^{-\operatorname{dim} X}[X]\right)$ and as

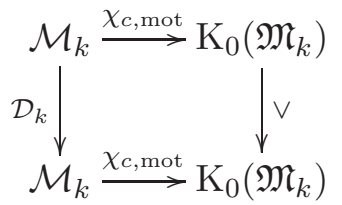

is commutative we conclude that $\left(\chi_{\operatorname{mot}}\langle X\rangle\right)^{\vee}=\mathbb{L}_{\text {mot }}^{-\operatorname{dim} X} \otimes \chi_{c, \operatorname{mot}}[X]$.

The rest of this section is devoted to the proof of Theorem 4.2. For this purpose we give a more convenient presentation of $\widetilde{\mathrm{K}}_{0}\left(\operatorname{Var}_{k}\right)$.

For the moment let $\widetilde{\mathrm{K}}_{0}^{\mathrm{bl}}\left(\operatorname{Var}_{k}\right)$ be the free abelian group on isomorphism classes of pairs of smooth varieties (denoted by $\langle X, Y\rangle_{\mathrm{bl}}$, where $\langle X\rangle_{\mathrm{bl}}$ stands for $\langle X, \emptyset\rangle_{\mathrm{bl}}$ ), with the same relations as for $\widetilde{\mathrm{K}}_{0}\left(\operatorname{Var}_{k}\right)$ except that we impose the excision relation only for blow-ups at smooth centers (as $\langle X, \emptyset\rangle_{\mathrm{bl}}=\langle X \sqcup Y, Y\rangle_{\mathrm{bl}}$ by blowing up $X \sqcup Y$ along $Y$ we get by additivity $\langle X \sqcup Y\rangle_{\mathrm{bl}}=\langle X\rangle_{\mathrm{bl}}+\langle Y\rangle_{\mathrm{bl}}$ and $\left.\langle\emptyset\rangle_{\mathrm{bl}}=0\right)$.

Proposition 4.4. The obvious group homomorphism $\widetilde{\mathrm{K}}_{0}^{\mathrm{bl}}\left(\operatorname{Var}_{k}\right) \longrightarrow \widetilde{\mathrm{K}}_{0}\left(\operatorname{Var}_{k}\right)$ is an isomorphism. In other words, the value group $\widetilde{\mathrm{K}}_{0}\left(\operatorname{Var}_{k}\right)$ of the universal Euler characteristic for pairs of $k$-varieties has a presentation as the free group on isomorphism classes of pairs of smooth $k$-varieties, modulo additivity, Gysin and excision for blow-ups at smooth centers.

The strategy for the proof of the proposition is to construct an inverse by defining a map $\Psi: \widehat{\mathrm{K}_{0}}\left(\operatorname{Var}_{k}\right) \longrightarrow \widetilde{\mathrm{K}}_{0}^{\mathrm{bl}}\left(\operatorname{Var}_{k}\right)$ that is the obvious map on a smooth pair $\{X, Y\}$ and factors through the Gysin relation. It is enough to define $\Psi\{X\}$ for all varieties $\{X\}$ and then to set $\Psi\{X, Y\}=$ $\Psi\{X\}-\Psi\{Y\}$. Then $\Psi$ will automatically fulfill additivity. For a smooth variety $X$ we want $\Psi\{X\}$ to be equal to $\langle X\rangle_{\mathrm{bl}}$.

We proceed by induction on the dimension. To be more precise we use that $\underline{\lim } \widehat{\mathrm{K}_{0}}\left(\operatorname{Var}_{k}\right)_{n} \cong$ $\widehat{\mathrm{K}_{0}}\left(\operatorname{Var}_{k}\right)$, where $\widehat{\mathrm{K}_{0}}\left(\operatorname{Var}_{k}\right)_{n}$ denotes the free abelian group on isomorphism classes of pairs of varieties of dimension at most $n$ modulo excision and additivity. In dimension zero we just set $\Psi\{X\}=\langle X\rangle_{\text {bl }}$. Suppose now that $\Psi$ has already been defined for pairs $\{X, Y\}$ with $\operatorname{dim} X<n$, that it factors through the excision relation for such pairs (in particular it is additive on disjoint unions in dimensions smaller than $n$ ) and that $\Psi\{X\}=\langle X\rangle_{\mathrm{bl}}$ for $X$ smooth and $\operatorname{dim} X<n$. In the following five steps we extend $\Psi$ to $\widehat{\mathrm{K}_{0}}\left(\operatorname{Var}_{k}\right)_{n}$.

Step 1. Suppose $f: X^{\prime} \longrightarrow X$ is proper with $Y^{\prime}=f^{-1} Y$, and suppose that $f$ induces an isomorphism $X^{\prime}-Y^{\prime} \cong X-Y$. Suppose $\operatorname{dim} X, \operatorname{dim} X^{\prime} \leqslant n$ and $\operatorname{dim} Y, \operatorname{dim} Y^{\prime}<n, X$ and $X^{\prime}$ smooth. Then $\left\langle X^{\prime}\right\rangle_{\mathrm{bl}}-\Psi\left\{Y^{\prime}\right\}=\langle X\rangle_{\mathrm{bl}}-\Psi\{Y\}$.

Proof. Suppose first that $X$ and $X^{\prime}$ are connected. Owing to the factorization theorem $f$ can be factored into a sequence of blow-ups and blow-downs with smooth centers disjoint from $X-Y$. So we can assume that $X^{\prime} \longrightarrow X$ is a blow-up along $Z \subset X$ a smooth closed subvariety with $Z \subset Y$. Let $E \subset Y^{\prime}$ be the exceptional divisor of this blow-up. We have $\left\langle X^{\prime}\right\rangle_{\mathrm{bl}}-\langle E\rangle_{\mathrm{bl}}=\langle X\rangle_{\mathrm{bl}}-\langle Z\rangle_{\mathrm{bl}}$ and furthermore $\Psi\left\{Y^{\prime}\right\}-\langle E\rangle_{\mathrm{bl}}=\Psi\{Y\}-\langle Z\rangle_{\mathrm{bl}}$ by the induction hypothesis. Hence $\left\langle X^{\prime}\right\rangle_{\mathrm{bl}}-\Psi\left\{Y^{\prime}\right\}=$ $\langle X\rangle_{\mathrm{bl}}-\Psi\{Y\}$.

Now in general $X$ can be written as the disjoint union of connected components $X_{1} \sqcup \cdots \sqcup X_{l} \sqcup$ $\cdots \sqcup X_{n}$ such that $Y_{i}:=X_{i} \cap Y$ is a proper subset of $X_{i}$ for $i \leqslant l$ and $Y_{i}=X_{i}$ for $i>l$. In particular $Y_{i}$ is smooth for $i>l$, and as $\operatorname{dim} Y_{i}<n$ the induction hypothesis yields $\Psi\left\{Y_{i}\right\}=\left\langle Y_{i}\right\rangle_{\mathrm{bl}}=\left\langle X_{i}\right\rangle_{\mathrm{bl}}$ 
and hence

$$
\langle X\rangle_{\mathrm{bl}}-\psi\{Y\}=\sum_{i=1}^{l}\left\langle X_{i}\right\rangle_{\mathrm{bl}}-\psi\left\{Y_{i}\right\} .
$$

Decomposing $X^{\prime}=X_{1}^{\prime} \sqcup \cdots \sqcup X_{l^{\prime}}^{\prime} \sqcup \cdots \sqcup X_{n^{\prime}}^{\prime}$ in the same way and setting $Y_{i}^{\prime}=X_{i}^{\prime} \cap Y$ yields

$$
\left\langle X^{\prime}\right\rangle_{\mathrm{bl}}-\psi\left\{Y^{\prime}\right\}=\sum_{i=1}^{l^{\prime}}\left\langle X_{i}^{\prime}\right\rangle_{\mathrm{bl}}-\psi\left\{Y_{i}^{\prime}\right\} .
$$

As $f$ induces an isomorphism $\bigsqcup_{i=1}^{l^{\prime}} X_{i}^{\prime}-Y_{i}^{\prime} \cong \bigsqcup_{i=1}^{l} X_{i}-Y_{i}$ it follows that $l=l^{\prime}$ and we may assume that $f$ induces $\left(X_{i}^{\prime}, Y_{i}^{\prime}\right) \longrightarrow\left(X_{i}, Y_{i}\right)$ for $i \leqslant l$, hence the two sums are equal.

Now let $X$ be an arbitrary $n$-dimensional variety. Choose $\pi: \widetilde{X} \longrightarrow X$ proper with $\tilde{X}$ smooth such that $\pi$ induces an isomorphism over the smooth locus of $X$. Let $S \subset X$ be the singular locus of $X$, and let $\widetilde{S} \subset \widetilde{X}$ be the inverse image of $S$. As we want $\Psi\{\widetilde{X}, \widetilde{S}\}$ to be equal to $\Psi\{X, S\}$ we set $\Psi\{X\}:=\langle\widetilde{X}\rangle_{\mathrm{bl}}-\Psi\{\widetilde{S}\}+\Psi\{S\}$ (the right-hand side has already been defined as $\widetilde{X}$ is smooth and $\operatorname{dim} S, \operatorname{dim} \widetilde{S}<n)$.

Since any two choices of $\widetilde{X} \longrightarrow X$ are dominated by a third one (take for example the closure of $X-S$ which is diagonally embedded in the product and resolve the singularities), Step 1 implies that $\langle\widetilde{X}\rangle_{\mathrm{bl}}-\Psi\{\widetilde{S}\}+\Psi\{S\}$ is independent of this choice. It is clear that $\Psi$ is additive with respect to disjoint unions and that for smooth $X$ we get $\Psi\{X\}=\langle X\rangle_{\mathrm{bl}}$.

We have to prove that $\Psi$ factors through excision for pairs of varieties of dimension $\leqslant n$.

Step 2. The map $\Psi$ factors through excision for $f:\left(X^{\prime}, Y^{\prime}\right) \longrightarrow(X, Y)$ with $X$ and $X^{\prime}$ both smooth of dimension $\leqslant n$.

Proof. Like in the proof of Step 1 we can decompose $X$ and $X^{\prime}$ into connected components. Now use $\Psi\left\{X_{i}\right\}=\left\langle X_{i}\right\rangle_{\mathrm{bl}}$ and $\Psi\left\{X_{i}^{\prime}\right\}=\left\langle X_{i}^{\prime}\right\rangle_{\mathrm{bl}}$ and apply Step 1 to the $X_{i}$ and $X_{i}^{\prime}$ with $i \leqslant l$.

Step 3. The map $\Psi$ factors through excision for $f:\left(X^{\prime}, Y^{\prime}\right) \longrightarrow(X, Y)$ with $\operatorname{dim} X, \operatorname{dim} X^{\prime} \leqslant n$, $X^{\prime}$ smooth and $\operatorname{dim} Y<n$.

Proof. Let $S \subset Y$ be the singular locus of $X$, let $\widetilde{X} \longrightarrow X$ be a resolution of singularities which is an isomorphism outside $S$, and let $\widetilde{Y}$ be the inverse image of $Y$ and $\widetilde{S}$ the inverse image of $S$. Then by definition $\Psi\{X, S\}=\Psi\{\widetilde{X}, \widetilde{S}\}$ and by induction $\Psi\{Y, S\}=\Psi\{\widetilde{Y}, \widetilde{S}\}$. Hence $\Psi\{X, Y\}=\Psi\{\widetilde{X}, \widetilde{Y}\}$.

Let $\widehat{X}$ be a resolution of singularities of the closure of $X-Y$ in $\widetilde{X} \times X^{\prime}$ (we embed $X-Y$ diagonally). We have a commutative diagram

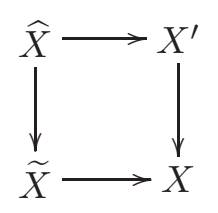

of proper birational maps which yield isomorphisms over $X-Y$. Let $\widehat{Y}$ be the inverse image of $Y$ in $\widehat{X}$. Then $\Psi\{\widehat{X}, \widehat{Y}\}=\Psi\left\{X^{\prime}, Y^{\prime}\right\}$ and $\Psi\{\widehat{X}, \widehat{Y}\}=\Psi\{\widetilde{X}, \widetilde{Y}\}$ as $\widehat{X}, \widetilde{X}$ and $X^{\prime}$ are smooth.

Step 4. The map $\Psi$ factors through excision for $f:\left(X_{1}, Y_{1}\right) \longrightarrow\left(X_{2}, Y_{2}\right)$ with $\operatorname{dim} X_{1}, \operatorname{dim} X_{2} \leqslant n$ and $\operatorname{dim} Y_{1}, \operatorname{dim} Y_{2}<n$.

Proof. For $i=1,2$ let $S_{i}$ be the singular locus of $X_{i}$, and let $Z_{i}=Y_{i} \cup S_{i}$. Then $f^{-1}\left(Z_{2}\right)=Z_{1}$ and $f$ induces an isomorphism of smooth varieties $X_{1}-Z_{1} \cong X_{2}-Z_{2}$ (as the singular locus of $X_{i}-Y_{i}$ is $S_{i}-Y_{i}$ ). Let $\widetilde{X}_{i}$ be a resolution of singularities of $X_{i}$ that is an isomorphism over $X_{i}-Z_{i}$ : so if $\widetilde{Z}_{i}$ is the preimage of $Z_{i}$ in $\widetilde{X}_{i}$ then $\widetilde{X}_{i}-\widetilde{Z}_{i} \cong X_{i}-Z_{i}$. Let $X_{3}$ be the closure of $X_{1}-Z_{1}$ 


\section{F. BitTneR}

(embedded diagonally) in $\widetilde{X_{1}} \times \widetilde{X_{2}}$. Let $\widetilde{X_{3}}$ a resolution of singularities of $X_{3}$ (that is an isomorphism over $X_{1}-Z_{1}$ ). We thus have a commutative diagram

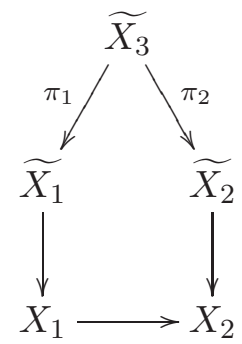

where $\pi_{1}^{-1}\left(\widetilde{Z_{1}}\right)=\pi_{2}^{-1}\left(\widetilde{Z_{2}}\right)=: \widetilde{Z_{3}}$ and $\pi_{1}, \pi_{2}$ induce isomorphisms outside $\widetilde{Z_{3}}$. As $\widetilde{X_{i}}$ are smooth for $1 \leqslant i \leqslant 3$ and $\operatorname{dim} Z_{1}, \operatorname{dim} Z_{2}<n$ we have

$$
\Psi\left\{X_{1}, Z_{1}\right\}=\Psi\left\{\widetilde{X_{1}}, \widetilde{Z_{1}}\right\}=\Psi\left\{\widetilde{X_{3}}, \widetilde{Z_{3}}\right\}=\Psi\left\{\widetilde{X_{2}}, \widetilde{Z_{2}}\right\}=\Psi\left\{X_{2}, Z_{2}\right\}
$$

The induction hypothesis gives

$$
\Psi\left\{Z_{1}, Y_{1}\right\}=\Psi\left\{Z_{2}, Y_{2}\right\} .
$$

Adding these two equations up yields $\Psi\left\{X_{1}, Y_{1}\right\}=\Psi\left\{X_{2}, Y_{2}\right\}$.

Step 5. The map $\Psi$ factors through excision for $f:\left(X^{\prime}, Y^{\prime}\right) \longrightarrow(X, Y)$ with $\operatorname{dim} X, \operatorname{dim} X^{\prime} \leqslant n$.

Proof. Let $X_{1}, \ldots, X_{l}$ be the irreducible components of $X$, and let $Y_{i}:=X_{i} \cap Y$. Let $B:=\bigcup_{Y_{i} \neq X_{i}} X_{i}$, and let $N:=Y \cap B$. Let $A=\bigcup_{Y_{i}=X_{i}} X_{i}$. Hence $X=A \cup B$ and $Y=A \cup N$.

Consider the proper map $A \sqcup B \longrightarrow X$ which induces an isomorphism $A \sqcup B-(A \cap B \sqcup A \cap$ $B) \cong X-(A \cap B)$. As $\operatorname{dim} A \cap B<n$ we get $\Psi\{A \sqcup B, A \cap B \sqcup A \cap B\}=\Psi\{X, A \cap B\}$, hence $\Psi\{A \sqcup B\}=\Psi\{X\}+\Psi\{A \cap B\}$.

Analogously we get $\Psi\{A \sqcup N\}=\Psi\{Y\}+\Psi\{A \cap N\}$.

Subtracting yields $\Psi\{A \sqcup B, A \sqcup N\}=\Psi\{X, Y\}$ (because $A \cap N=A \cap B \cap Y=A \cap B$ ).

On the other hand $\Psi\{A \sqcup B, A \sqcup N\}=\Psi\{B, N\}$, hence $\Psi\{B, N\}=\Psi\{X, Y\}$.

The same reasoning yields $\Psi\left\{B^{\prime}, N^{\prime}\right\}=\Psi\left\{X^{\prime}, Y^{\prime}\right\}$ (with analogous notations).

Restricting $f$ yields a proper map $\left(B^{\prime}, N^{\prime}\right) \longrightarrow(B, N)$ which induces an isomorphism $B^{\prime}-N^{\prime} \cong$ $B-N$, and as $\operatorname{dim} N, \operatorname{dim} N^{\prime}<n$ this finishes the proof.

This finishes the induction step.

As the Gysin relation holds in $\widetilde{\mathrm{K}}_{0}^{\mathrm{bl}}\left(\operatorname{Var}_{k}\right)$, we get an induced homomorphism $\bar{\Psi}: \widetilde{\mathrm{K}}_{0}\left(\operatorname{Var}_{k}\right) \longrightarrow$ $\widetilde{\mathrm{K}}_{0}^{\mathrm{bl}}\left(\operatorname{Var}_{k}\right)$ which can inductively be seen to be an inverse of $\widetilde{\mathrm{K}}_{0}^{\mathrm{bl}}\left(\operatorname{Var}_{k}\right) \longrightarrow \widetilde{\mathrm{K}}_{0}\left(\operatorname{Var}_{k}\right)$. This establishes Proposition 4.4.

We now prove Theorem 4.2 by defining an inverse $\chi: \widetilde{\mathrm{K}}_{0}\left(\operatorname{Var}_{k}\right) \longrightarrow \mathrm{K}_{0}\left(\operatorname{Var}_{k}\right)$ on classes of smooth varieties and checking compatibility with excision for blow-ups and the Gysin relation. Let $X$ be a smooth connected variety. Choose a smooth completion $\bar{X} \supset X$ of $X$ with $D=\bar{X}-X$ a divisor with simple normal crossings.

Step 1. The element $\sum(-\mathbb{L})^{l}\left[D^{(l)}\right]$ of $\mathrm{K}_{0}\left(\operatorname{Var}_{k}\right)$ is independent of the choice of the completion of $X$ (we denote it by $\chi(X)$ ).

Proof. In view of the weak factorization theorem we only have to compare the result for a completion $X \subset \bar{X}$ and the completion $X \subset \mathrm{Bl}_{Z} \bar{X}=: \bar{X}^{\prime}$, with $Z$ a smooth and irreducible closed subvariety of $\bar{X}$ disjoint from $X$ which has normal crossings with $D=\bar{X}-X$.

Let $D_{0}$ be an irreducible component of $D$ containing $Z$ and let $\left\{D_{i}\right\}_{i \in I}$ be the remaining irreducible components. Then the irreducible components of $D^{\prime}=\bar{X}^{\prime}-X$ are $D_{i}^{\prime}=\mathrm{Bl}_{Z \cap D_{i}} D_{i}$ 


\section{The universal Euler CHARACTERISTiC}

(where $i \in\{0\} \cup I$ ) and the exceptional divisor $E$ of the blow-up. For $K \subset\{0\} \cup I$ we put $D_{K}:=\bigcap_{j \in K} D_{j}$ (where $D_{\emptyset}$ is understood to be $\bar{X}$ ), $D_{K}^{\prime}:=\bigcap_{j \in K} D_{j}^{\prime}, Z_{K}:=Z \cap D_{K}$ and $E_{K}:=E \cap D_{K}^{\prime}$. Denote the codimension of $Z_{K}$ in $D_{K}$ by $d_{K}$. As $Z$ has normal crossings with $D$ we get $D_{K}^{\prime}=\mathrm{Bl}_{Z_{K}} D_{K}$ with exceptional divisor $E_{K}$, hence by Lemma 3.5 we have

$$
\left[D_{K}^{\prime}\right]-\mathbb{L}\left[E_{K}\right]=\left[D_{K}\right]-\mathbb{L}^{d_{K}}\left[Z_{K}\right] .
$$

Denote by $E^{(l)}$ the inverse image of $E$ in $D^{\prime(l)}$. For $l=0, \ldots, n$ the preceding equality yields

$$
\begin{aligned}
\mathbb{L}^{l}\left[D^{\prime(l)}\right] & =\mathbb{L}^{l} \sum_{|K|=l}\left[D_{K}^{\prime}\right]+\mathbb{L}^{l} \sum_{|K|=l-1}\left[E_{K}\right] \\
& =\mathbb{L}^{l} \sum_{|K|=l}\left(\left[D_{K}\right]+\mathbb{L}\left[E_{K}\right]-\mathbb{L}^{d_{K}}\left[Z_{K}\right]\right)+\mathbb{L}^{l} \sum_{|K|=l-1}\left[E_{K}\right] \\
& =\mathbb{L}^{l}\left[D^{(l)}\right]+\mathbb{L}^{l+1}\left[E^{(l)}\right]-\sum_{|K|=l} \mathbb{L}^{l+d_{K}}\left[Z_{K}\right]+\mathbb{L}^{l}\left[E^{(l-1)}\right]
\end{aligned}
$$

(for $l=0$ the last term is zero).

For $K \subset I$ we get $Z_{K \cup\{0\}}=Z_{K}$ and hence $d_{K \cup\{0\}}=d_{K}-1$, which yields

$$
\mathbb{L}^{|K \cup\{0\}|+d_{K \cup\{0\}}}\left[Z_{K \cup\{0\}}\right]=\mathbb{L}^{|K|+d_{K}}\left[Z_{K}\right]
$$

and

$$
\sum_{K \subset I \cup\{0\}}(-1)^{|K|} \mathbb{L}^{|K|+d_{K}}\left[Z_{K}\right]=0 .
$$

Taking the alternating sum then yields

$$
\sum(-\mathbb{L})^{l}\left[D^{\prime(l)}\right]=\sum(-\mathbb{L})^{l}\left[D^{(l)}\right]
$$

as claimed.

Step 2. For a smooth $X$ with connected components $X_{i}$ we set $\chi(X):=\sum \chi\left(X_{i}\right)$.

Step 3. Let $X$ be a smooth variety, and let $Z \subset X$ be a smooth closed subvariety. Let $E$ be the exceptional divisor of $\mathrm{Bl}_{Z} X \longrightarrow X$. Then $\chi\left(\mathrm{Bl}_{Z} X, E\right)=\chi(X, Z)$.

Proof. By decomposing $X$ and $Z$ into connected components we see that it suffices to prove the claim for connected $X$ and $Z$. Denote the dimension of $X$ by $n$. Choose a smooth completion $\bar{X}$ of $X$ such that $D=\bar{X}-X$ is a divisor with simple normal crossings and $\bar{Z}$ is smooth and has normal crossings with $D$. So in particular $D \cap \bar{Z} \subset \bar{Z}$ is a simple normal crossings divisor. Then $\mathrm{Bl}_{\bar{Z}} \bar{X}$ is a smooth completion of $\mathrm{Bl}_{Z} X$ with $D^{\prime}:=\mathrm{Bl}_{\bar{Z}} \bar{X}-\mathrm{Bl}_{Z} X$ a normal crossings divisor. The exceptional divisor $\bar{E}$ of this blow-up is a smooth completion of $E$ with $\bar{E}-E=\bar{E} \cap D^{\prime}$ a simple normal crossings divisor. Denote by $E^{(l)}$ the preimage of $\bar{E}$ in $D^{\prime(l)}$ and by $Z^{(l)}$ the preimage of $\bar{Z}$ in $D^{(l)}$. Now

$$
\begin{aligned}
\chi\left(\mathrm{Bl}_{Z} X\right) & =\left[D^{\prime(0)}\right]-\mathbb{L}\left[D^{\prime(1)}\right]+\cdots+(-\mathbb{L})^{n}\left[D^{\prime(n)}\right], \\
\chi(E) & =\left[E^{(0)}\right]-\mathbb{L}\left[E^{(1)}\right]+\cdots+(-\mathbb{L})^{n}\left[E^{(n)}\right], \\
\chi(X) & =\left[D^{(0)}\right]-\mathbb{L}\left[D^{(1)}\right]+\cdots+(-\mathbb{L})^{n}\left[D^{(n)}\right], \\
\chi(Z) & =\left[Z^{(0)}\right]-\mathbb{L}\left[Z^{(1)}\right]+\cdots+(-\mathbb{L})^{n}\left[Z^{(n)}\right] .
\end{aligned}
$$

As $D^{\prime(l)}-E^{(l)} \cong D^{(l)}-Z^{(l)}$ we get $\left[D^{\prime(l)}\right]-\left[E^{(l)}\right]=\left[D^{(l)}\right]-\left[Z^{(l)}\right]$ in $\mathrm{K}_{0}\left(\operatorname{Var}_{k}\right)$ and consequently

$$
\chi\left(\mathrm{Bl}_{Z} X, E\right)=\chi\left(\mathrm{Bl}_{Z} X\right)-\chi(E)=\chi(X)-\chi(Z)=\chi(X, Z) .
$$




\section{F. BitTneR}

Step 4. For $X$ smooth and connected and $D \subset X$ a smooth divisor, we have $\chi(X-D)=\chi(X)-$ $\mathbb{L} \chi(D)$.

Proof. Let $\bar{X} \supset X$ be a smooth completion of $X$ such that $D_{1}:=(\bar{X}-X) \cup \bar{D}$ is a simple normal crossings divisor, where $\bar{D}$ denotes the closure of $D$ in $\bar{X}$. Let $D_{2}:=\bar{X}-X$. Denote by $\Delta^{(l)}$ the inverse image of $\bar{D}$ in $D_{2}^{(l)}$. Then $\chi(D)=\sum(-\mathbb{L})^{l}\left[\Delta^{(l)}\right]$ and on the other hand

$$
\chi(X-D)=\sum(-\mathbb{L})^{l}\left[D_{1}^{(l)}\right]=\sum(-\mathbb{L})^{l}\left[D_{2}^{(l)}\right]-\mathbb{L} \sum(-\mathbb{L})^{l}\left[\Delta^{(l)}\right] .
$$

Step 5. For $X$ smooth and connected, we have $\chi\left(\mathbb{P}^{1} \times X,\{\infty\} \times X\right)=\mathbb{L} \chi(X)$.

Proof. Let $\bar{X}$ be a smooth completion of $X$ with $D=\bar{X}-X$ a simple normal crossings divisor. Then $\mathbb{P}^{1} \times \bar{X}$ is a smooth completion of $\mathbb{P}^{1} \times X$ such that the complement of $\mathbb{P}^{1} \times X$ is $\mathbb{P}^{1} \times D$, a simple normal crossings divisor. This yields

$$
\begin{aligned}
\chi\left(\mathbb{P}^{1} \times X,\{\infty\} \times X\right) & =\chi\left(\mathbb{P}^{1} \times X\right)-\chi(X) \\
& =\sum(-\mathbb{L})^{l}\left(\left[\left(\mathbb{P}^{1} \times D\right)^{(l)}\right]-\left[D^{(l)}\right]\right) \\
& =\mathbb{L} \chi(X) .
\end{aligned}
$$

Hence $\chi$ factors through $\widetilde{\mathrm{K}}_{0}\left(\operatorname{Var}_{k}\right)$. We will now denote the induced mapping by $\chi$.

Step 6 . Let $X$ be a smooth connected variety. Then $\chi_{c} \chi\langle X\rangle=\langle X\rangle$ in $\widetilde{\mathrm{K}}_{0}\left(\operatorname{Var}_{k}\right)$.

Proof. Let $\bar{X} \supset X$ be a smooth completion with $D:=\bar{X}-X$ a simple normal crossings divisor. Let $D_{1}, \ldots, D_{n}$ be the irreducible components of $D$. We proceed by induction on $n$.

If $X$ is complete then obviously $\chi_{c} \chi\langle X\rangle=\langle X\rangle$. This establishes the claim for $n=0$.

Let $X=X_{0} \subset X_{1} \subset \cdots \subset X_{n}=\bar{X}$ be given by $X_{i}:=X_{i+1}-\left(D_{i+1} \cap X_{i+1}\right)$. Then

$$
\begin{aligned}
\chi_{c} \chi\langle X\rangle & =\chi_{c} \chi\left\langle X_{1}-\left(D_{1} \cap X_{1}\right)\right\rangle \\
& =\chi_{c} \chi\left\langle X_{1}\right\rangle-\chi_{c} \chi\left\langle\mathbb{P}^{1} \times\left(D_{1} \cap X_{1}\right),\{\infty\} \times\left(D_{1} \cap X_{1}\right)\right\rangle
\end{aligned}
$$

by the Gysin relation. Now $X_{1} \subset \bar{X}$ is a smooth completion with $\bar{X}-X_{1}$ a simple normal crossings divisor with less then $n$ irreducible components. The same holds for $D_{1} \cap X_{1} \subset D_{1}$ and for $\mathbb{P}^{1} \times$ $\left(D_{1} \cap X_{1}\right) \subset \mathbb{P}^{1} \times D_{1}$. Hence by induction we get

$$
\begin{aligned}
\chi_{c} \chi & \left\langle X_{1}\right\rangle-\chi_{c} \chi\left\langle\mathbb{P}^{1} \times\left(D_{1} \cap X_{1}\right),\{\infty\} \times\left(D_{1} \cap X_{1}\right)\right\rangle \\
& =\chi_{c} \chi\left\langle X_{1}\right\rangle-\chi_{c} \chi\left\langle\mathbb{P}^{1} \times\left(D_{1} \cap X_{1}\right)\right\rangle+\chi_{c} \chi\left\langle\{\infty\} \times\left(D_{1} \cap X_{1}\right)\right\rangle \\
& =\left\langle X_{1}\right\rangle-\left\langle\mathbb{P}^{1} \times\left(D_{1} \cap X_{1}\right)\right\rangle+\left\langle\{\infty\} \times\left(D_{1} \cap X_{1}\right)\right\rangle \\
& =\langle X\rangle
\end{aligned}
$$

again by the Gysin relation.

Step 7. The mappings $\chi_{c}$ and $\chi$ are mutually inverse.

Proof. Obviously $\chi \chi_{c}[X]=[X]$ for $X$ smooth and complete, and we already know that $\chi_{c} \chi\langle X\rangle=$ $\langle X\rangle$ for $X$ smooth.

Step 8. For $X$ a smooth and connected variety the image of $\chi\langle X\rangle$ in $\mathcal{M}_{k}$ is equal to $\mathcal{D}_{k}\left(\mathbb{L}^{-\operatorname{dim} X}[X]\right)$ $=\mathbb{L}^{\operatorname{dim} X} \mathcal{D}_{k}([X])$.

Proof. Let $(\bar{X}, D)$ be a smooth simple normal crossings completion. Then

$$
\mathcal{D}_{k}([X])=\sum(-1)^{l} \mathcal{D}_{k}\left(\left[D^{(l)}\right]\right)=\sum(-1)^{l} \mathbb{L}^{l-\operatorname{dim} X}\left[D^{(l)}\right] .
$$

This finishes the proof of Theorem 4.2 . 


\section{The Universal Euler CHARACTERISTIC}

\section{Relative Grothendieck groups of varieties}

Let $S$ be a (not necessarily irreducible) variety over $k$. Let $\mathrm{K}_{0}\left(\operatorname{Var}_{S}\right)$ be the free abelian group on isomorphism classes $[X]_{S}$ of varieties $X$ over $S$ where we impose the relations $[X]_{S}=[X-Y]_{S}+$ $[Y]_{S}$ for $Y \subset X$ a closed subvariety (hence again $[\emptyset]_{S}=0$ ). We call it the Grothendieck group of $S$-varieties.

This group, too, has simple presentations.

Theorem 5.1. The Grothendieck group of $S$-varieties has the following alternative presentations:

$\mathrm{sm})$ as the abelian group generated by the isomorphism classes of $S$-varieties which are smooth over $k$ subject to the relations $[X]_{S}=[X-Y]_{S}+[Y]_{S}$, where $X$ is smooth and $Y \subset X$ is a smooth closed subvariety;

bl) as the abelian group generated by the isomorphism classes of $S$-varieties which are smooth over $k$ and proper over $S$ subject to the relations $[\emptyset]_{S}=0$ and $\left[\mathrm{Bl}_{Y} X\right]_{S}-[E]_{S}=[X]_{S}-[Y]_{S}$, where $X$ is smooth over $k$ and proper over $S, Y \subset X$ is a closed smooth subvariety, $\mathrm{Bl}_{Y} X$ is the blow-up of $X$ along $Y$ and $E$ is the exceptional divisor of this blow-up.

Moreover, we get the same group if in case $(\mathrm{sm})$ we restrict to varieties which are quasi-projective over $S$ or if in case (bl) we restrict to varieties which are projective over $S$. We can also restrict to connected varieties in both presentations.

We proceed in the same way as in the absolute case. For the moment denote by $\mathrm{K}_{0}^{\mathrm{sm}}\left(\operatorname{Var}_{S}\right)$ the free abelian group on isomorphism classes $[X]_{S, \mathrm{sm}}$ of varieties over $S$ which are smooth over $k$, modulo $[X]_{S, \mathrm{sm}}=[X-Y]_{S, \mathrm{sm}}+[Y]_{S, \mathrm{sm}}$ for $X \subset Y$ a smooth closed subvariety of $X$.

Step 1. The group homomorphism $\mathrm{K}_{0}^{\mathrm{sm}}\left(\operatorname{Var}_{S}\right) \longrightarrow \mathrm{K}_{0}\left(\operatorname{Var}_{S}\right)$ is an isomorphism.

Proof. We use the same stratification argument as in the absolute case.

Again we can restrict to smooth connected varieties in the presentation ( $\mathrm{sm}$ ) by decomposing into connected components.

We can also stratify by smooth varieties which are quasi-projective over $S$.

Now we establish the presentation $(\mathrm{bl})$. Denote by $\mathrm{K}_{0}^{\mathrm{bl}}\left(\operatorname{Var}_{S}\right)$ the free abelian group on isomorphism classes of varieties $[X]_{S, \mathrm{bl}}$, where $X$ is a variety which is proper over $S$ and smooth over $k$, modulo $[X]_{S, \mathrm{bl}}-[Y]_{S, \mathrm{bl}}=\left[\mathrm{Bl}_{Y} X\right]_{S, \mathrm{bl}}-[E]_{S, \mathrm{bl}}$ for $Y$ a closed smooth subvariety of $X$ and $E$ the exceptional divisor of the blow-up of $X$ along $Y$ and $[\emptyset]_{S, \text { bl }}=0$ (blowing up along $Y$ yields then $\left.[X \sqcup Y]_{S, \mathrm{bl}}=[X]_{S, \mathrm{bl}}+[Y]_{S, \mathrm{bl}}\right)$.

Decomposing into connected components and noting that the blow-up along a disjoint union is the successive blow-up along the connected components one sees that this can also be described as the free abelian group on isomorphism classes $[X]_{S, \text { bl }}$ of smooth complete irreducible varieties with imposed relations $[\emptyset]_{S, \mathrm{bl}}=0$ and $\left[\mathrm{Bl}_{Y} X\right]_{S, \mathrm{bl}}-[E]_{S, \mathrm{bl}}=[X]_{S, \mathrm{bl}}-[Y]_{S, \mathrm{bl}}$, where $Y \subset X$ is a closed irreducible smooth subvariety.

Step 2. The group homomorphism $\mathrm{K}_{0}^{\mathrm{bl}}\left(\operatorname{Var}_{S}\right) \longrightarrow \mathrm{K}_{0}\left(\operatorname{Var}_{S}\right)$ which maps $[X]_{S, \text { bl }}$ to $[X]_{S}$ is an isomorphism.

Proof. For an arbitrary variety $X$ over $S$ there exist a proper $\bar{X} \rightarrow S$ and an open dense immersion $X \hookrightarrow \bar{X}$ over $S$. If $X$ is smooth over $k$ and connected we can even find $\bar{X}$ smooth with $\bar{X}-X$ a simple normal crossings divisor (by resolution of singularities and principalization). As the weak factorization theorem also works over a base variety (note that a birational map between two irreducible varieties which are proper over $S$ is automatically proper) we can construct an inverse in the same way as in the absolute case. 


\section{F. BITTNER}

Using the fact that we can restrict to generators which are quasi-projective over $S$ in the presentation ( $\mathrm{sm}$ ) and that a connected smooth variety which is quasi-projective over $S$ has a smooth simple normal crossings completion which is projective over $S$ we see that we can restrict to generators projective over $S$ in the description (bl).

\section{The six operations}

The product of varieties makes $\mathrm{K}_{0}\left(\operatorname{Var}_{S}\right)$ a $\mathrm{K}_{0}\left(\operatorname{Var}_{k}\right)$-module. Let $\mathcal{M}_{k}$ denote $\mathrm{K}_{0}\left(\operatorname{Var}_{k}\right)\left[\mathbb{L}^{-1}\right]$, and let $\mathcal{M}_{S}$ denote the localization $\mathrm{K}_{0}\left(\operatorname{Var}_{S}\right)\left[\mathbb{L}^{-1}\right]$. Denote the class $[S]_{S}$ by $\mathbb{1}_{S}$.

Remark 6.1. If $f: S \longrightarrow S^{\prime}$ is a morphism of $k$-varieties, composition with $f$ yields a $\mathrm{K}_{0}\left(\operatorname{Var}_{k}\right)$ linear mapping $f_{!}: \mathrm{K}_{0}\left(\operatorname{Var}_{S}\right) \longrightarrow \mathrm{K}_{0}\left(\operatorname{Var}_{S^{\prime}}\right)$, hence we get an $\mathcal{M}_{k^{-}}$-linear mapping $f_{!}: \mathcal{M}_{S} \longrightarrow \mathcal{M}_{S^{\prime}}$. Pulling back along $f$ yields a $\mathrm{K}_{0}\left(\operatorname{Var}_{k}\right)$-linear mapping $f^{*}: \mathrm{K}_{0}\left(\operatorname{Var}_{S^{\prime}}\right) \longrightarrow \mathrm{K}_{0}\left(\operatorname{Var}_{S}\right)$ and hence an $\mathcal{M}_{k}$-linear mapping $\mathcal{M}_{S^{\prime}} \longrightarrow \mathcal{M}_{S}$.

Taking products yields a $\mathrm{K}_{0}\left(\operatorname{Var}_{k}\right)$-bilinear associative exterior product

$$
\otimes: \mathrm{K}_{0}\left(\operatorname{Var}_{S}\right) \times \mathrm{K}_{0}\left(\operatorname{Var}_{T}\right) \longrightarrow \mathrm{K}_{0}\left(\operatorname{Var}_{S \times T}\right)
$$

and hence an $\mathcal{M}_{k}$-bilinear associative map

$$
\otimes: \mathcal{M}_{S} \times \mathcal{M}_{T} \longrightarrow \mathcal{M}_{S \times T} .
$$

For morphisms $f: S \longrightarrow S^{\prime}$ and $g: T \longrightarrow T^{\prime}$ of varieties we get the identities $(f \times g) !(A \otimes B)=$ $f_{!}(A) \otimes g_{!}(B)$ and $(f \times g)^{*}(C \otimes D)=f^{*}(C) \otimes g^{*}(D)$. If $p: S \times S^{\prime} \longrightarrow S$ is the projection to the first factor we get $p^{*}(A)=A \otimes \mathbb{1}_{S^{\prime}}$ for $A \in \mathcal{M}_{S}$.

Remark 6.2. Taking fiber products yields an internal $\mathrm{K}_{0}\left(\operatorname{Var}_{k}\right)$-bilinear symmetric associative product $\otimes: \mathrm{K}_{0}\left(\operatorname{Var}_{S}\right) \times \mathrm{K}_{0}\left(\operatorname{Var}_{S}\right) \longrightarrow \mathrm{K}_{0}\left(\operatorname{Var}_{S}\right)$ and hence an $\mathcal{M}_{k}$-bilinear symmetric associative map $\otimes: \mathcal{M}_{S} \times \mathcal{M}_{S} \longrightarrow \mathcal{M}_{S}$ which provides $\mathcal{M}_{S}$ with the structure of a commutative $\mathcal{M}_{k}$-algebra.

This satisfies $f^{*}(C \otimes D)=f^{*}(C) \otimes f^{*}(D)$ and $\mathbb{1}_{S} \otimes A=A \otimes \mathbb{1}_{S}=A$.

For $A \in \mathcal{M}_{S^{\prime}}$ and $B \in \mathcal{M}_{S}$ we have $f_{!}\left(f^{*} A \otimes B\right)=A \otimes f_{!} B$. In other words, if we regard $\mathcal{M}_{S}$ as an $\mathcal{M}_{S^{\prime}}$-module via $f^{*}$, then $f_{\text {! }}$ is an $\mathcal{M}_{S^{\prime}}$-module homomorphism.

For $A, B \in \mathcal{M}_{S}$ and $C, D \in \mathcal{M}_{T}$ we get

$$
(A \otimes C) \otimes(B \otimes D)=(A \otimes B) \otimes(C \otimes D),
$$

hence the exterior product provides $\mathcal{M}_{S \times T}$ with the structure of an $\mathcal{M}_{S} \otimes \mathcal{M}_{T}$-algebra.

Definition 6.3. The duality involution relative to $S$ is the map defined as follows: There is a morphism $\mathcal{D}_{S}: \mathrm{K}_{0}\left(\operatorname{Var}_{S}\right) \longrightarrow \mathcal{M}_{S}$ which sends a generator $[X]_{S}$ with $X$ connected and smooth over $k$, proper over $S$, to $\mathbb{L}^{-\operatorname{dim} X}[X]_{S}$. A relative version of Lemma 3.5 shows that this is indeed compatible with blow-up relations. For $A \in \mathrm{K}_{0}\left(\operatorname{Var}_{k}\right)$ and $B \in \mathrm{K}_{0}\left(\operatorname{Var}_{S}\right)$ we get $\mathcal{D}_{S}(A B)=\mathcal{D}_{k}(A) \mathcal{D}_{S}(B)$ (this is easily checked on smooth proper generators), so $\mathcal{D}_{S}$ can be extended to a $\mathcal{D}_{k}$-linear morphism $\mathcal{D}_{S}: \mathcal{M}_{S} \longrightarrow \mathcal{M}_{S}$.

Indeed $\mathcal{D}_{S}$ is an involution. We have already noted in $\S 4$ that $\mathcal{D}_{k}(\mathbb{L})=\mathbb{L}^{-1}$ and that $\mathcal{D}_{k}$ is a lift of the duality morphism on the Grothendieck ring of Chow motives. For $A \in \mathcal{M}_{S}$ and $B \in \mathcal{M}_{T}$ we get

$$
\mathcal{D}_{S \times T}(A \otimes B)=\mathcal{D}_{S}(A) \otimes \mathcal{D}_{T}(B) .
$$

Definition 6.4. For $f: S \longrightarrow S^{\prime}$ define $f^{!}:=\mathcal{D}_{S} f^{*} \mathcal{D}_{S^{\prime}}$ and $f_{*}=\mathcal{D}_{S^{\prime}} f_{!} \mathcal{D}_{S}$.

These are both $\mathcal{M}_{k}$-linear mappings. 


\section{The universal Euler CHARACTERISTiC}

Remark 6.5. If $f$ is a proper mapping then $f_{*}$ coincides with $f_{!}$. If $f$ is an open embedding then $f^{!}$coincides with $f^{*}$. More generally, if $f$ is smooth of relative dimension $m$, then for $A \in \mathcal{M}_{S^{\prime}}$ we have $f^{*} A=\mathbb{L}^{m} f^{!} A$.

Proposition 6.6. Given a cartesian diagram as follows

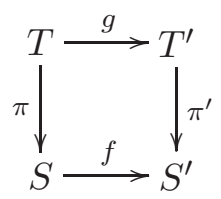

we have $g_{!} \pi^{*}=\pi^{*} f_{!}$and $g_{*} \pi^{!}=\pi^{\prime !} f_{*}$. If $f$ is proper or $\pi^{\prime}$ is smooth, then $g_{*} \pi^{*}=\pi^{*} f_{*}$.

Proof. The only thing to prove is $g_{*} \pi^{*}=\pi^{\prime *} f_{*}$ for $\pi^{\prime}$ smooth. So suppose $\pi^{\prime}$ is smooth of relative dimension $m$, hence the same holds for $\pi$.

Let $A \in \mathcal{M}_{S}$. Then due to Remark 6.5

$$
\pi^{\prime *} f_{*} A=\mathbb{L}^{m} \pi^{\prime !} f_{*} A=\mathbb{L}^{m} g_{*} \pi^{!} A=g_{*}\left(\mathbb{L}^{m} \pi^{!} A\right)=g_{*} \pi^{*} A .
$$

Definition 6.7. Define the dualizing element of $\mathcal{M}_{S}$ by $\mathbb{D}_{S}:=\mathcal{D}_{S}\left(\mathbb{1}_{S}\right)$. For $A, B \in \mathcal{M}_{S}$ define

$$
\operatorname{Hom}(A, B):=\mathcal{D}_{S}\left(A \otimes \mathcal{D}_{S} B\right) .
$$

This yields a mapping $\mathcal{M}_{S} \times \mathcal{M}_{S} \longrightarrow \mathcal{M}_{S}$ which is $\mathcal{D}_{k}$-linear in the first and $\mathcal{M}_{k}$-linear in the second component. For $A \in \mathcal{M}_{S}$ we get $\operatorname{Hom}\left(A, \mathbb{D}_{S}\right)=\mathcal{D}_{S}(A)$.

List of properties 6.8. Let $f: S \longrightarrow S^{\prime}$ be a morphism of $k$-varieties. Then the following identities hold:

$$
\begin{aligned}
\operatorname{Hom}\left(A, f_{*} B\right) & =f_{*} \operatorname{Hom}\left(f^{*} A, B\right) \text { for } A \in \mathcal{M}_{S^{\prime}} \text { and } B \in \mathcal{M}_{S}, \\
\operatorname{Hom}\left(f^{*} A, f^{!} B\right) & =f^{!} \operatorname{Hom}(A, B) \text { for } A, B \in \mathcal{M}_{S^{\prime}}, \\
f_{*} \operatorname{Hom}\left(A, f^{!} B\right) & =\operatorname{Hom}\left(f_{!} A, B\right) \text { for } A \in \mathcal{M}_{S} \text { and } B \in \mathcal{M}_{S^{\prime}} .
\end{aligned}
$$

For $A, B, C \in \mathcal{M}_{S}$ we get

$$
\operatorname{Hom}(A, \operatorname{Hom}(B, C))=\operatorname{Hom}(A \otimes B, C) .
$$

For $p: S \times S^{\prime} \longrightarrow S$ the projection to the first factor and for $A, B \in \mathcal{M}_{S}$ we have

$$
\operatorname{Hom}\left(p^{*} A, p^{*} B\right)=p^{*} \operatorname{Hom}(A, B) \text {. }
$$

Proof. We have

$$
\begin{aligned}
f_{*} \operatorname{Hom}\left(f^{*} A, B\right) & =f_{*} \mathcal{D}_{S}\left(f^{*} A \otimes \mathcal{D}_{S} B\right)=\mathcal{D}_{S^{\prime}} f_{!}\left(f^{*} A \otimes \mathcal{D}_{S} B\right)=\mathcal{D}_{S^{\prime}}\left(A \otimes f_{!} \mathcal{D}_{S} B\right) \\
& =\mathcal{D}_{S^{\prime}}\left(A \otimes \mathcal{D}_{S^{\prime}} f_{*} B\right)=\operatorname{Hom}\left(A, f_{*} B\right), \\
\operatorname{Hom}\left(f^{*} A, f^{!} B\right) & =\mathcal{D}_{S}\left(f^{*} A \otimes \mathcal{D}_{S} f^{!} B\right)=\mathcal{D}_{S}\left(f^{*} A \otimes f^{*} \mathcal{D}_{S^{\prime}} B\right)=\mathcal{D}_{S} f^{*}\left(A \otimes \mathcal{D}_{S^{\prime}} B\right) \\
& =f^{!} \mathcal{D}_{S^{\prime}}\left(A \otimes \mathcal{D}_{S^{\prime}} B\right)=f^{!} \operatorname{Hom}(A, B), \\
f_{*} \operatorname{Hom}\left(A, f^{!} B\right) & =f_{*} \mathcal{D}_{S}\left(A \otimes \mathcal{D}_{S} f^{!} B\right)=f_{*} \mathcal{D}_{S}\left(A \otimes f^{*} \mathcal{D}_{S^{\prime}} B\right) \\
& =\mathcal{D}_{S^{\prime}} f_{!}\left(A \otimes f^{*} \mathcal{D}_{S^{\prime}} B\right)=\mathcal{D}_{S^{\prime}}\left(f_{!} A \otimes \mathcal{D}_{S^{\prime}} B\right)=\operatorname{Hom}\left(f_{!} A, B\right) .
\end{aligned}
$$

Furthermore,

$$
\begin{aligned}
\operatorname{Hom}(A, \operatorname{Hom}(B, C)) & =\operatorname{Hom}\left(A, \mathcal{D}_{S}\left(B \otimes \mathcal{D}_{S} C\right)\right) \\
& =\mathcal{D}_{S}\left(A \otimes\left(B \otimes \mathcal{D}_{S} C\right)\right)=\operatorname{Hom}(A \otimes B, C) .
\end{aligned}
$$




\section{F. BITTNER}

Finally, using $\mathcal{D}_{S \times S^{\prime}}(C \otimes D)=\mathcal{D}_{S}(C) \otimes \mathcal{D}_{S^{\prime}}(D)$ for $C \in \mathcal{M}_{S}$ and $D \in \mathcal{M}_{S^{\prime}}$ we get

$$
\begin{aligned}
\operatorname{Hom}\left(p^{*} A, p^{*} B\right) & =\mathcal{D}_{S \times S^{\prime}}\left(\left(A \otimes \mathbb{1}_{S^{\prime}}\right) \otimes \mathcal{D}_{S \times S^{\prime}}\left(B \otimes \mathbb{1}_{S^{\prime}}\right)\right) \\
& =\mathcal{D}_{S \times S^{\prime}}\left(\left(A \otimes \mathbb{1}_{S^{\prime}}\right) \otimes\left(\mathcal{D}_{S} B \otimes \mathbb{D}_{S^{\prime}}\right)\right) \\
& =\mathcal{D}_{S \times S^{\prime}}\left(\left(A \otimes \mathcal{D}_{S} B\right) \otimes \mathbb{D}_{S^{\prime}}\right)=\mathcal{D}_{S}\left(A \otimes \mathcal{D}_{S} B\right) \otimes \mathcal{D}_{S^{\prime}}\left(\mathbb{D}_{S^{\prime}}\right) \\
& =\operatorname{Hom}(A, B) \otimes \mathbb{1}_{S^{\prime}}=p^{*} \operatorname{Hom}(A, B) .
\end{aligned}
$$

\section{Equivariant Grothendieck groups of varieties}

Let $G$ be a finite group. There are also $G$-equivariant versions of Grothendieck groups of varieties:

Let $\mathrm{K}_{0}^{\prime G}\left(\operatorname{Var}_{k}\right)$ be the free abelian group on $G$-isomorphism classes $[G \circlearrowright X]$ (or shorthand $[X]$ ) of varieties $X$ with a good $G$-action over $k$, divided out by the relations $[X]=[X-Y]+[Y]$ for $Y \subset X$ a closed $G$-invariant subvariety. The product of varieties (with diagonal $G$-action) induces a commutative ring structure on $\mathrm{K}_{0}^{\prime G}\left(\operatorname{Var}_{k}\right)$. Now note that a linear automorphism of a vector bundle $V \longrightarrow X$ induces the identity on the (singular) cohomology of $\mathbb{P}(V)$ as it induces the identity on the cohomology of the fibers. If $G$ acts on $X$ and $V$ is trivial and carries a linear $G$-action over the action on $X$, this action factors as a $G$-action over the base and the $G$-action on the base only. As the Grothendieck groups cannot distinguish between trivial and non-trivial bundles, this serves as a motivation to define $\mathrm{K}_{0}^{G}\left(\operatorname{Var}_{k}\right)$ as $\mathrm{K}_{0}^{\prime G}\left(\operatorname{Var}_{k}\right)$ modulo the subgroup $M_{k}^{G}$ generated by expressions of the form $[G \circlearrowright \mathbb{P}(V)]-\left[\mathbb{P}^{n} \times(G \circlearrowright X)\right]$, where $X$ is a variety with good $G$-action, $V$ is a vector bundle of rank $n+1$ over $X$ with linear $G$-action over the action on $X, G \circlearrowright \mathbb{P}(V)$ denotes the projectivization of this action and $\mathbb{P}^{n} \times(G \circlearrowright X)$ denotes the action of $G$ on the right factor only. In fact this subgroup is an ideal in $\mathrm{K}_{0}^{\prime G}\left(\operatorname{Var}_{k}\right)$.

Note that as for a vector bundle $V \longrightarrow X$ of rank $n$ we have $[G \circlearrowright V]=\left[G \circlearrowright \mathbb{P}\left(V \oplus \mathcal{O}_{X}\right)\right]-[G \circlearrowright$ $\mathbb{P}(V)]$ we get the equality $[G \circlearrowright V]=\left[\mathbb{A}^{n} \times(G \circlearrowright X)\right]$ in $\mathrm{K}_{0}^{G}\left(\operatorname{Var}_{k}\right)$.

LEMma 7.1. The group $\mathrm{K}_{0}^{\prime G}\left(\operatorname{Var}_{k}\right)$ has the following alternative presentations:

$\mathrm{sm})$ as the abelian group generated by the isomorphism classes of smooth varieties with good $G$-action subject to the relations $[X]=[X-Y]+[Y]$, where $X$ is smooth with good $G$-action and $Y \subset X$ is a smooth $G$-invariant closed subvariety;

bl) as the abelian group generated by the isomorphism classes of smooth complete varieties with good $G$-action subject to the relations $[\emptyset]=0$ and $\left[\mathrm{Bl}_{Y} X\right]-[E]=[X]-[Y]$, where $X$ is a smooth complete variety with good $G$-action, $Y \subset X$ is a closed smooth $G$-invariant subvariety, $\mathrm{Bl}_{Y} X$ is the blow-up of $X$ along $Y$ and $E$ is the exceptional divisor of this blow-up.

Moreover, we get the same group if in case $(\mathrm{sm})$ we restrict to quasi-projective varieties or if in case (bl) we restrict to projective varieties. In both presentations we can also restrict to varieties such that $G$ acts transitively on the connected components.

Proof. Stratifying by smooth $G$-invariant equidimensional varieties establishes the presentation (sm). We can also stratify by quasi-projective $G$-invariant varieties. We can decompose further into smooth varieties such that $G$ acts transitively on the connected components.

Now note that a smooth connected variety $X$ with a good $G$-action has a good smooth completion with as complement a $G$-invariant simple normal crossings divisor: Take a normal completion $\overline{X / G}$ of $X / G$ and take the normalization $\tilde{X}$ of $\overline{X / G}$ in $X$. By construction $\tilde{X}$ carries a good $G$-action (induced by the $G$-action on $k(X))$ extending the $G$-action on $X$ which it contains as an open and dense subvariety. Furthermore $\widetilde{X}$ is finite over $\overline{X / G}$ and hence complete. An equivariant resolution of singularities of $\widetilde{X}$ making the complement of $X$ a simple normal crossings divisor then gives a completion $\bar{X}$ with the desired properties. Actually as in Remark 2.7 it suffices that $G$ acts 


\section{The universal Euler CHARACTERISTiC}

transitively on the connected components of $X$. A reasoning slightly different from before then establishes the presentation (bl) as we will show in Lemma 7.2. Restricting to quasi-projective varieties in the presentation ( $\mathrm{sm}$ ) shows that we can restrict to projective varieties in (bl) (here we can take any smooth projective equivariant simple normal crossings completion, as on a projective variety a $G$-action is automatically good).

Lemma 7.2. Denote by $\mathrm{K}_{0}^{\prime G, \mathrm{bl}}\left(\operatorname{Var}_{k}\right)$ the free abelian group on isomorphism classes $[X]_{\mathrm{bl}}$ of smooth complete varieties $X$ over $k$ with a good $G$-action, modulo the relations for blow-ups along smooth closed $G$-invariant subvarieties. Then the group homomorphism $\mathrm{K}_{0}^{\prime G \text {,bl }}\left(\operatorname{Var}_{k}\right) \longrightarrow \mathrm{K}_{0}^{\prime G}\left(\operatorname{Var}_{k}\right)$ which sends $[X]_{\mathrm{bl}}$ to $[X]$ is an isomorphism.

Proof. We construct an inverse, but rather than writing it down explicitly we use the fact that $\mathrm{K}_{0}^{\prime G}\left(\operatorname{Var}_{k}\right) \cong \lim \mathrm{K}_{0}^{\prime G}\left(\operatorname{Var}_{k}\right)_{n}$, where $\mathrm{K}_{0}^{\prime G}\left(\operatorname{Var}_{k}\right)_{n}$ denotes the free abelian group on isomorphism classes of smooth varieties with a good $G$-action of dimension at most $n$ modulo the relations for smooth closed $G$-invariant subvarieties. (The expression used in the case without group action may not be the right one - take for example $\mathbb{P}^{2}$ with the involution interchanging two coordinates $x_{1}$ and $x_{2}$ and consider this as the completion of the complement of the two divisors $\left\{x_{1}=0\right\}$ and $\left\{x_{2}=0\right\}$.)

We have a morphism $\chi_{c, 0}: \mathrm{K}_{0}^{\prime G}\left(\operatorname{Var}_{k}\right)_{0} \longrightarrow \mathrm{K}_{0}^{\prime G, \mathrm{bl}}\left(\operatorname{Var}_{k}\right)$ by just sending $[X]$ to $[X]_{\mathrm{bl}}$.

Now let $n>0$. Suppose that for $m<n$ we have already defined compatible morphisms $\chi_{c, m}$ : $\mathrm{K}_{0}^{\prime G}\left(\operatorname{Var}_{k}\right)_{m} \longrightarrow \mathrm{K}_{0}^{\prime G, \mathrm{bl}}\left(\operatorname{Var}_{k}\right)$ in such a way that $\chi_{c, m}[X]=[X]_{\mathrm{bl}}$ for $X$ (smooth and) complete.

Suppose $X$ is smooth of dimension at most $n$, and suppose $G$ acts transitively on the connected components of $X$. We choose an equivariant simple normal crossings completion $\bar{X}$. For $D=\bar{X}-X$ we denote by $D^{\circ,(l)}$ the open $l$-fold intersections of $D$ (we remove all $(l+1$ )-fold intersections from each $l$-fold intersection), such that $\bar{X}=\bigcup_{l \geqslant 0} D^{\circ,(l)}$.

Consider the expression $[\bar{X}]_{\mathrm{bl}}-\sum_{l=1}^{n} \chi_{c, n-1}\left[D^{\circ,(l)}\right]$. It does not depend on the chosen completion: owing to the factorization theorem we only need to compare it to the corresponding expression for $\bar{X}^{\prime}=\mathrm{Bl}_{Z} \bar{X}$, where $Z \subseteq D$ is a smooth $G$-invariant closed subvariety of $\bar{X}$ which has normal crossings with $D$. Then $\bar{D}^{\prime}=\bar{X}^{\prime}-X$ is equal to $E \cup \widetilde{D}$, where $E$ is the exceptional divisor (which is smooth and not necessarily connected) and $\widetilde{D}$ is the proper transform of $D$. For $l>0$ this yields $D^{\prime o,(l)} \cap E=E \cap \widetilde{D}^{\circ,(l-1)}$ with complement $\widetilde{D}^{\circ,(l)}-E \cap \widetilde{D}^{\circ,(l)}$. Hence

$$
\chi_{c, n-1}\left[D^{\prime \circ,(l)}\right]=\chi_{c, n-1}\left[E \cap \widetilde{D}^{\circ,(l-1)}\right]+\chi_{c, n-1}\left[\widetilde{D}^{\circ,(l)}-E \cap \widetilde{D}^{\circ,(l)}\right],
$$

and as $\widetilde{D}^{\circ,(l)}-E \cap \widetilde{D}^{\circ,(l)} \cong D^{\circ,(l)}-Z \cap D^{\circ,(l)}$ and thus

$$
\chi_{c, n-1}\left[\widetilde{D}^{\circ,(l)}-E \cap \widetilde{D}^{\circ,(l)}\right]=\chi_{c, n-1}\left[D^{\circ,(l)}\right]-\chi_{c, n-1}\left[Z \cap D^{\circ,(l)}\right],
$$

we find

$$
\begin{aligned}
{\left[\bar{X}^{\prime}\right]_{\mathrm{bl}} } & -\sum_{l=1}^{n} \chi_{c, n-1}\left[D^{\prime \circ,(l)}\right] \\
= & {\left[\bar{X}^{\prime}\right]_{\mathrm{bl}}-\sum_{l=1}^{n} \chi_{c, n-1}\left[D^{\circ,(l)}\right]+\sum_{l=1}^{n} \chi_{c, n-1}\left[Z \cap D^{\circ,(l)}\right]-\sum_{l=1}^{n} \chi_{c, n-1}\left[E \cap \widetilde{D}^{\circ,(l-1)}\right] . }
\end{aligned}
$$

Now $\sum_{l=1}^{n} \chi_{c, n-1}\left[Z \cap D^{\circ,(l)}\right]=\chi_{c, n-1}[Z]=[Z]_{\mathrm{bl}}$ and $\sum_{l=1}^{n} \chi_{c, n-1}\left[E \cap \widetilde{D}^{\circ,(l-1)}\right]=[E]_{\mathrm{bl}}$, and as $\left[\bar{X}^{\prime}\right]_{\mathrm{bl}}-[E]_{\mathrm{bl}}+[Z]_{\mathrm{bl}}=[X]_{\mathrm{bl}}$ we get

$$
\left[\bar{X}^{\prime}\right]_{\mathrm{bl}}-\sum_{l=1}^{n} \chi_{c, n-1}\left[D^{\prime \circ,(l)}\right]=[\bar{X}]_{\mathrm{bl}}-\sum_{l=1}^{n} \chi_{c, n-1}\left[D^{\circ,(l)}\right] .
$$




\section{F. BitTneR}

Hence we can define $e_{n}(X)$ as $[\bar{X}]_{\mathrm{bl}}-\sum_{l=1}^{n} \chi_{c, n-1}\left[D^{\circ,(l)}\right]$. By construction $e_{n}(X)=[X]_{\mathrm{bl}}$ if $X$ is complete and $e_{n}(X)=\chi_{c, n-1}[X]$ if $X$ is of dimension less than $n$. We still have to check that for a smooth closed $G$-invariant subvariety $Y$ of $X$ (such that $G$ acts transitively on the connected components of $Y)$ the equality $e_{n}(X)=e_{n}(X-Y)+e_{n}(Y)$ holds. As in the case without group action we choose $\bar{X} \supset X$ smooth and complete such that $D=\bar{X}-X$ is a simple normal crossings divisor and such that the closure $\bar{Y}$ of $Y$ in $\bar{X}$ is also smooth and has normal crossings with $D$ (and such that everything is compatible with the group action). Again we take $\widetilde{X}=\mathrm{Bl}_{\bar{Y}} \bar{X}$ as completion of $X-Y$. Then $\widetilde{X}-(X-Y)=\widetilde{D} \cup E$, where $E$ is the exceptional divisor and $\widetilde{D}$ is the proper transform of $D$. As $\widetilde{D}^{\circ,(l)}-E \cap \widetilde{D}^{\circ,(l)} \cong D^{\circ,(l)}-\bar{Y} \cap D^{\circ,(l)}$ we get

$$
\begin{aligned}
e_{n}(X-Y) & =[\tilde{X}]_{\mathrm{bl}}-\sum_{l=1}^{n} \chi_{c, n-1}\left(\left[E \cap \widetilde{D}^{\circ,(l-1)}\right]\right)-\sum_{l=1}^{n} \chi_{c, n-1}\left[\widetilde{D}^{\circ,(l)}-E \cap \widetilde{D}^{\circ,(l)}\right] \\
& =[\tilde{X}]_{\mathrm{bl}}-[E]_{\mathrm{bl}}-\sum_{l=1}^{n} \chi_{c, n-1}\left[D^{\circ,(l)}\right]+\sum_{l=1}^{n} \chi_{c, n-1}\left[\bar{Y} \cap D^{\circ,(l)}\right] \\
& =[\bar{X}]_{\mathrm{bl}}-[\bar{Y}]_{\mathrm{bl}}-\sum_{l=1}^{n} \chi_{c, n-1}\left[D^{\circ,(l)}\right]+\sum_{l=1}^{n} \chi_{c, n-1}\left[\bar{Y} \cap D^{\circ,(l)}\right] \\
& =e_{n}(X)-e_{n}(Y) .
\end{aligned}
$$

Hence $e_{n}$ induces a morphism $\chi_{c, n}: \mathrm{K}_{0}^{\prime G}\left(\operatorname{Var}_{k}\right)_{n} \longrightarrow \mathrm{K}_{0}^{\prime G, \mathrm{bl}}\left(\operatorname{Var}_{k}\right)$ which is compatible with $\chi_{n-1}$.

We can also consider varieties over a base variety $S$ with good $G$-action over $S$ (by this we mean that $X \rightarrow S$ is $G$-equivariant where we give $S$ the trivial $G$-action). Denote by $\mathrm{K}_{0}^{\prime G}\left(\operatorname{Var}_{S}\right)$ the free group on isomorphism classes $[X]_{S}$ of such varieties modulo relations for closed subvarieties. It has the structure of a $\mathrm{K}_{0}^{\prime G}\left(\operatorname{Var}_{k}\right)$-module provided by the product of varieties with diagonal $G$-action. We define $\mathrm{K}_{0}^{G}\left(\operatorname{Var}_{S}\right)$ to be $\mathrm{K}_{0}^{\prime G}\left(\operatorname{Var}_{S}\right)$ modulo the submodule $M_{S}^{G}$ generated as a group by expressions of the form $[G \circlearrowright \mathbb{P}(V)]_{S}-\left[\mathbb{P}^{n} \times(G \circlearrowright X)\right]_{S}$ (so in particular $\mathrm{K}_{0}^{G}\left(\operatorname{Var}_{S}\right)$ is a $\mathrm{K}_{0}^{G}\left(\operatorname{Var}_{k}\right)$-module). Again for a vector bundle $V \longrightarrow X$ of rank $n$ we have $[G \circlearrowright V]_{S}=\left[\mathbb{A}^{n} \times(G \circlearrowright X)\right]_{S}$ in $\mathrm{K}_{0}^{G}\left(\operatorname{Var}_{S}\right)$.

By the same reasoning as above we get the following lemma.

Lemma 7.3. The group $\mathrm{K}_{0}^{\prime G}\left(\operatorname{Var}_{S}\right)$ has the following alternative presentations:

$\mathrm{sm})$ as the abelian group generated by the isomorphism classes of $S$-varieties with good $G$-action over $S$ which are smooth over $k$ subject to the relations $[X]_{S}=[X-Y]_{S}+[Y]_{S}$, where $X$ is smooth and $Y \subset X$ is a smooth closed $G$-invariant subvariety;

bl) as the abelian group generated by the isomorphism classes of $S$-varieties with good $G$-action over $S$ which are smooth over $k$ and proper over $S$ subject to the relations $[\emptyset]_{S}=0$ and $\left[\mathrm{Bl}_{Y} X\right]_{S}-[E]_{S}=[X]_{S}-[Y]_{S}$, where $X$ is smooth over $k$ and proper over $S$ and carries a good $G$-action over $S, Y \subset X$ is a closed smooth $G$-invariant subvariety, $\mathrm{Bl}_{Y} X$ is the blow-up of $X$ along $Y$ and $E$ is the exceptional divisor of this blow-up.

Moreover, we get the same group if in case $(\mathrm{sm})$ we restrict to varieties which are quasi-projective over $S$ or if in case (bl) we restrict to varieties which are projective over $S$. We can also restrict to varieties such that $G$ acts transitively on the connected components in both presentations.

The $\mathrm{K}_{0}^{\prime G}\left(\operatorname{Var}_{k}\right)$-submodule $M_{S}^{G}$ has convenient generators (as an abelian group). First an auxiliary lemma is needed.

Lemma 7.4. Let $X$ be a smooth irreducible variety with a good $G$-action. Let $V \rightarrow X$ be a vector bundle with linear $G$-action over the action on $X$. Let $X \subset \bar{X}$ be open and dense such that the $G$-action extends to a good action on $\bar{X}$. Then there is a non-empty open subvariety $U$ of $X$ and 


\section{The universal Euler CHARACTERISTiC}

an equivariant $\pi: X^{\prime} \longrightarrow \bar{X}$, projective over $\bar{X}$, such that $X^{\prime}$ is smooth, $\pi$ induces an isomorphism over $U$ and $X^{\prime}$ carries a vector bundle with $G$-action extending $\left.V\right|_{U}$.

Before we prove the lemma we make some remarks on Grassmann bundles. Suppose $V \longrightarrow X$ is a vector bundle of rank $n \geqslant r$. Then there is the Grassmann variety $p: \operatorname{Grass}_{r}(V) \longrightarrow X$ which parametrizes quotients of rank $r$ or equivalently sub-bundles of rank $n-r$ : there is a short exact sequence

$$
0 \longrightarrow S \longrightarrow p^{*} V \longrightarrow Q \longrightarrow 0
$$

where $S$ is the universal sub-bundle and $Q$ is the universal quotient bundle, such that for a morphism $f: Y \longrightarrow X$ there is a one-to-one correspondence between liftings $\phi$ of $f$ to $\operatorname{Grass}_{r}(V)$ and subbundles $S_{\phi}$ of $f^{*} V$ which is given by $\phi \mapsto \phi^{*} S$. Now suppose we are given an action of a finite group $G$ on $X$ and an action of $G$ on $V$ which is linear over the action on $X$. This induces an action of $G$ on $\operatorname{Grass}_{r}(V)$ where naively speaking $g \in G$ sends an $r$-codimensional subspace $S_{x}$ of $E_{x}$ to $g S_{x} \subseteq E_{g x}$. With respect to this action $p$ is equivariant, and $p^{*} E$ carries an induced (diagonal) $G$-action which is linear over the action on $\operatorname{Grass}_{r}(V)$. This action preserves $S$ and hence induces an action on $Q$.

Now for a $G$-equivariant morphism $f: Y \longrightarrow X$ there is a one-to-one correspondence between $G$-equivariant liftings $\phi$ of $f$ to $\operatorname{Grass}_{r}(V)$ and $r$-codimensional $G$-sub-bundles $S_{\phi}$ of $f^{*} V$, given by $\phi \mapsto \phi^{*} S$. Then also $f^{*} V / S_{\phi} \cong \phi^{*} Q$ with the induced $G$-action.

Proof of Lemma 7.4. Let $K=k(X)$ the function field of $X$. Then over the generic point of $X$ the vector bundle $V$ is given by a $K$-vector space $W$ with an additive $G$-action such that $g(\lambda w)=$ $g(\lambda) g(w)$ for $\lambda \in K$ and $w \in W$. We choose a $K$-basis $w_{1}, \ldots, w_{r}$ of $W$.

As the action of $G$ on $\bar{X}$ is good, $\bar{X}$ can be covered by affine open subschemes $\operatorname{Spec}(B)$, where $B \subset K$ is preserved by $G$. Consider the free $B$-module on pairs $(g, i)$ with $g \in G$ and $1 \leqslant i \leqslant r$. This module carries a linear $G$-action over the action on $B$ by setting $h(\lambda(g, i))=h(\lambda)(h g, i)$. The action glues to a $G$-action on the trivial bundle $\mathbb{A} \frac{|G| r}{X}$, linear over the action on $\bar{X}$.

Consider the $G$-equivariant $K$-linear surjection from the $K$-vector space on pairs $(g, i)$ to $W$ which sends $(g, i)$ to $g\left(w_{i}\right)$. Over an open $G$-invariant subvariety $U \subset X$ this extends to a $G$-equivariant surjection of the trivial bundle to $V$.

Consider the $r$ th Grassmannian $\operatorname{Grass}_{r}\left(\mathbb{A}_{\frac{1}{X}}{ }^{\mid}\right)$of $\mathbb{A} \frac{|G| r}{X}$ which classifies $r$-dimensional quotients. It carries a $G$-action, and the universal quotient bundle carries a linear $G$-action over this action. Over $U$ we get a $G$-equivariant section provided by $V$ such that pulling back the universal quotient bundle to $U$ yields $\left.V\right|_{U}$ with the original $G$-action.

We can now take the closure $\widetilde{X}$ of this section in $\operatorname{Grass}_{r}\left(\mathbb{A}_{\bar{X}}^{|G| r}\right)$ and the restriction of the universal quotient bundle to $\widetilde{X}$. Taking a $G$-equivariant resolution $X^{\prime}$ of singularities for $\tilde{X}$ and pulling back this bundle we are done.

Lemma 7.5. The subgroup $M_{S}^{G}$ of $\mathrm{K}_{0}^{\prime G}\left(\operatorname{Var}_{S}\right)$ is generated by expressions of the form $[G \circlearrowright \mathbb{P}(V)]_{S}-$ $\left[\mathbb{P}^{n} \times(G \circlearrowright X)\right]_{S}$, where $X$ is a smooth variety, projective over $S$, with good $G$-action, transitive on the connected components, $V$ is a vector bundle of rank $n+1$ over $X$ with a linear $G$-action over $X$, and $G \circlearrowright \mathbb{P}(V)$ denotes the action induced by the action on $V$.

Proof. Let $X$ be an arbitrary variety over $S$ with good $G$-action. Suppose $V$ is a vector bundle of rank $n+1$ with a linear $G$-action over the action on $X$. We proceed by induction on the dimension of $X$.

If $X$ is zero dimensional, by decomposing it into $G$-orbits we can write the difference $[G \circlearrowright$ $\mathbb{P}(V)]_{S}-\left[\mathbb{P}^{n} \times(G \circlearrowright X)\right]_{S}$ as a linear combination of the generators described above. 


\section{F. BITTNER}

For the induction step we choose a smooth $G$-invariant open subvariety $W$, quasi-projective over $S$, such that the complement has smaller dimension. The induction hypothesis shows that it suffices to write $\left[G \circlearrowright \mathbb{P}\left(\left.V\right|_{W}\right)\right]_{S}-\left[\mathbb{P}^{n} \times(G \circlearrowright W)\right]_{S}$ as a linear combination of the described generators. Decomposing further we can assume that $G$ acts transitively on the connected components of $W$.

Identifying $\left.V\right|_{W}$ with $G \times{ }^{H}\left(\left.V\right|_{W_{0}}\right)$, where $W_{0}$ is a connected component of $W$ and $H$ is its stabilizer, we may assume that $W$ is connected.

We now choose a smooth $\bar{W} \supset W$, projective over $S$, such that the $G$-action extends to $\bar{W}$ and $W$ is dense in $\bar{W}$. The preceding lemma and the induction hypothesis show that there is an open non-empty $U \subset W$ such that $\left[G \circlearrowright \mathbb{P}\left(\left.V\right|_{U}\right)\right]_{S}-\left[\mathbb{P}^{n} \times(G \circlearrowright U)\right]_{S}$ can be written as a linear combination of the desired generators. Hence applying the induction hypothesis to $W-U$ we are done.

Summarizing we get the following theorem.

THEOREM 7.6. The ring $\mathrm{K}_{0}^{G}\left(\operatorname{Var}_{k}\right)$ is the free abelian group on smooth projective (respectively, complete) varieties with good $G$-action (transitive on the connected components), with the product described above, modulo blow-up relations and the subgroup generated by expressions of the form $[G \circlearrowright \mathbb{P}(V)]-\left[\mathbb{P}^{n} \times(G \circlearrowright X)\right]$, where $X$ is a smooth projective variety with a $G$-action transitive on the connected components, and $V \longrightarrow X$ is a vector bundle of rank $n+1$ over $X$ with a linear action over the action on $X$.

More generally the next theorem is obtained.

ThEOREM 7.7. The group $\mathrm{K}_{0}^{G}\left(\operatorname{Var}_{S}\right)$ is the free abelian group on smooth varieties, projective (respectively, proper) over $S$ with (good) $G$-action over $S$ (transitive on the connected components), modulo blow-up relations and the subgroup generated by expressions of the form $[G \circlearrowright \mathbb{P}(V)]_{S}-\left[\mathbb{P}^{n} \times(G \circlearrowright\right.$ $X)]_{S}$, where $X$ is a smooth variety, projective over $S$, with a $G$-action transitive on the connected components, and $V \longrightarrow X$ is a vector bundle of rank $n+1$ over $X$ with a linear action over the action on $X$.

\section{Operations in the equivariant setting}

Let $\varphi: G \longrightarrow H$ be a morphism of finite groups. This induces a restriction homomorphism

$$
\operatorname{Res}_{G}^{H}: \mathrm{K}_{0}^{H}\left(\operatorname{Var}_{S}\right) \longrightarrow \mathrm{K}_{0}^{G}\left(\operatorname{Var}_{S}\right)
$$

which in the case $S=$ Spec $k$ is a ring homomorphism.

On the other hand the product of varieties with diagonal $G$-action yields a $\mathrm{K}_{0}^{G}\left(\operatorname{Var}_{k}\right)$-module structure on $\mathrm{K}_{0}^{G}\left(\operatorname{Var}_{S}\right)$. Hence $\mathrm{K}_{0}^{G}\left(\operatorname{Var}_{S}\right)$ carries the structure of a $\mathrm{K}_{0}\left(\operatorname{Var}_{k}\right)$-module.

Let $\mathcal{M}_{k}^{G}$ denote $\mathrm{K}_{0}^{G}\left(\operatorname{Var}_{k}\right)\left[\mathbb{L}^{-1}\right]$, and let $\mathcal{M}_{S}^{G}$ denote the localization $\mathrm{K}_{0}^{G}\left(\operatorname{Var}_{S}\right)\left[\mathbb{L}^{-1}\right]$.

As the morphism $\operatorname{Res}_{G}^{H}$ is $\mathrm{K}_{0}\left(\operatorname{Var}_{k}\right)$-linear it induces an $\mathcal{M}_{k}$-linear morphism $\operatorname{Res}_{G}^{H}: \mathcal{M}_{S}^{H} \longrightarrow$ $\mathcal{M}_{S}^{G}$.

For a group homomorphism $\varphi: G \longrightarrow H$ there is also an induction morphism

$$
\operatorname{Ind}_{G}^{H}: \mathrm{K}_{0}^{G}\left(\operatorname{Var}_{S}\right) \longrightarrow \mathrm{K}_{0}^{H}\left(\operatorname{Var}_{S}\right)
$$

which sends the class of a $G$-variety $X$ to the class of $H \times^{G} X$. (This factors through $\mathrm{K}_{0}^{G}\left(\operatorname{Var}_{S}\right)$ as, for a vector bundle $V \longrightarrow X$ with a linear $G$-action over an action on $X$, we have $H \times{ }^{G} \mathbb{P}(V)=$ $\mathbb{P}\left(H \times{ }^{G} V\right)$, where $H \times{ }^{G} V$ is a vector bundle over $H \times{ }^{G} X$ with a linear $H$-action over the action on the base.)

The induction morphism $\operatorname{Ind}_{G}^{H}$ is $\mathrm{K}_{0}\left(\operatorname{Var}_{k}\right)$-linear and hence induces an $\mathcal{M}_{k}$-linear morphism $\operatorname{Ind}_{G}^{H}: \mathcal{M}_{S}^{G} \longrightarrow \mathcal{M}_{S}^{H}$. 


\section{The universal Euler CHARACTERISTiC} $\mathcal{M}_{k}^{H}$.

Restriction and induction are related by the fact that $\operatorname{Ind}_{G}^{H} \operatorname{Res}_{G}^{H}$ is multiplication with $[H / G] \in$

We define $f_{\text {! }}$ and $f^{*}$ as before.

Taking products yields an $\mathcal{M}_{k}$-bilinear associative map

$$
\otimes: \mathcal{M}_{S}^{G} \times \mathcal{M}_{T}^{H} \longrightarrow \mathcal{M}_{S \times T}^{G \times H} .
$$

In the case $G=H$ the diagonal mapping $G \longrightarrow G \times G$ induces an $\mathcal{M}_{k}^{G}$-bilinear associative map

$$
\nabla: \mathcal{M}_{S}^{G} \times \mathcal{M}_{T}^{G} \longrightarrow \mathcal{M}_{S \times T}^{G}
$$

Taking fiber products yields an $\mathcal{M}_{k}$-bilinear associative map

$$
\otimes: \mathcal{M}_{S}^{G} \times \mathcal{M}_{S}^{H} \longrightarrow \mathcal{M}_{S}^{G \times H}
$$

(which can also be obtained from the external product by pulling back along the diagonal map $S \longrightarrow S \times S)$. In the case $G=H$ the diagonal mapping induces an $\mathcal{M}_{k}^{G}$-bilinear associative map

$$
\otimes: \mathcal{M}_{S}^{G} \times \mathcal{M}_{S}^{G} \longrightarrow \mathcal{M}_{S}^{G}
$$

Using the presentation in Theorem 7.7 we see that we get a group homomorphism $\mathrm{K}_{0}^{G}\left(\operatorname{Var}_{S}\right) \longrightarrow$ $\mathcal{M}_{S}^{G}$ mapping the class of a smooth $X$ which is proper over $S$ with $G$ acting transitively on the connected components to $\mathbb{L}^{-\operatorname{dim} X}[X]_{S}$, which extends to a $\mathcal{D}_{k}^{G}$-linear morphism

$$
\mathcal{D}_{S}^{G}: \mathcal{M}_{S}^{G} \longrightarrow \mathcal{M}_{S}^{G} \text {. }
$$

For $A \in \mathcal{M}_{S}^{G}$ and $B \in \mathcal{M}_{T}^{H}$ we get

$$
\mathcal{D}_{S \times T}^{G \times H}(A \otimes B)=\mathcal{D}_{S}^{G}(A) \otimes \mathcal{D}_{T}^{H}(B) .
$$

We define $f^{!}, f_{*}$ and Hom as before and get equivariant versions of the identities in Section 6 .

\section{ACKnowledgements}

I am indebted to Eduard Looijenga, my thesis advisor. I would like to thank Jarosław Włodarczyk for answering my questions about the weak factorization theorem, and Jarosław Wiśniewski and the Mathematical Department of the University of Warsaw for their kind hospitality during my stay as a guest of the Polish EAGER node. I would also like to thank Jochen Heinloth for helpful discussions. I thank the referee for careful reading and many valuable comments and corrections.

\section{REFERENCES}

AKMW02 D. Abramovich, K. Karu, K. Matsuki and J. Włodarczyk, Torification and factorization of birational maps, J. Amer. Math. Soc. 15 (2002), 531-572.

Bon02 L. Bonavero, Factorisation faible des applications birationelles (d'après Abramovich, Karu, Matsuki, Wtodarczyk et Morelli], Séminaire Bourbaki, vol. 2000/2001, Astérisque 282 (2002), $1-37$.

DL01 J. Denef and F. Loeser, Geometry on arc spaces of algebraic varieties, in European Congress of Mathematics, Barcelona, 10-14 July 2000, Progress in Mathematics, vol. 201 (Birkhäuser, Basel, 2001), pp. 327-348.

GS96 H. Gillet and C. Soulé, Descent, motives and K-theory, J. reine angew. Math. 478 (1996), $127-176$.

GNA02 F. Guillén and V. Navarro Aznar, Un critère d'extension des foncteurs définis sur les schémas lisses, Publ. Math. Inst. Hautes Études Sci. 95 (2002), 1-91. 


\section{The UNIVERSAL EUler CHARACTERISTIC}

LL03 M. Larsen and V. A. Lunts, Motivic measures and stable birational geometry, Mosc. Math. J. 3 (2003), 85-95, 259.

Loo02 E. Looijenga, Motivic measures, Séminaire Bourbaki, vol. 1999/2000, Astérisque 276 (2002), $267-297$.

Sch94 A. Scholl, Classical motives, in Motives, Seattle, WA, 1991, Proc. Sympos. Pure Math., vol. 55I (American Mathematical Society, Providence, RI, 1994), 163-187.

Wło03 J. Włodarczyk, Toroidal varieties and the weak factorization theorem, Invent. Math. 154 (2003), 223-331.

Franziska Bittner franziska.bittner@uni-essen.de

Mathematisch Instituut, Universiteit Utrecht, The Netherlands

Current address: Universität Essen, FB 6, Mathematik, 45117 Essen, Germany 